\title{
Quantum dot integrated LEDs using photonic and excitonic color conversion
}

\author{
Hilmi Volkan Demir ${ }^{a, b, *}$, Sedat Nizamoglu ${ }^{b}$, Talha Erdem ${ }^{b}$, Evren Mutlugun ${ }^{a, b}$, \\ Nikolai Gaponikc, Alexander Eychmüller ${ }^{c, *}$
}

\author{
${ }^{a}$ Luminous! Center of Excellence for Semiconductor Lighting and Displays, School of Electrical and Electronic Engineering, School \\ of Physical and Mathematical Sciences, Nanyang Technological University, Singapore 639798, Singapore \\ b UNAM-Institute of Materials Science and Nanotechnology, Departments of Electrical and Electronics Engineering and Physics, \\ Bilkent University, Ankara 06800, Turkey \\ c Department of Physical Chemistry, TU Dresden, Bergstrasse 66b, D-01062 Dresden, Germany
}

Received 31 August 2011; received in revised form 21 October 2011; accepted 23 October 2011

Available online 23 November 2011

\author{
KEYWORDS \\ Quantum dot (QD); \\ Nanocrystal; \\ Light-emitting diode \\ (LED); \\ Förster resonance \\ energy transfer \\ (FRET); \\ Color conversion; \\ Solid state lighting
}

\begin{abstract}
Summary This review summarizes advantages, recent progress and challenges related to the quickly evolving research field of colloidal quantum dot integrated LEDs based on color conversion. We start with presenting a short general introduction to the field of solid state lighting and color conversion phenomena, which are essential for defining the requirements for highquality general lighting. Subsequently we continue with a brief description of the synthesis of nanocrystal quantum dots and their optical properties together with the advantages of utilizing them in LEDs as color convertors. Following this basic background, we review the recent efforts on quantum dot integrated color-conversion LEDs, Förster resonance energy transfer (FRET) converted LEDs and FRET-enhanced LEDs. Finally, we conclude with a future outlook on semiconductor lighting and quantum dot integrated LEDs.
\end{abstract}

(c) 2011 Elsevier Ltd. All rights reserved.

\section{Introduction}

One of the possible means to combat and prevent an irreversible climate change is to reduce the atmosphere pollution caused by the traditional energy production technologies. This may be achieved by increasing contributions of alternative energy sources and their diversification as

\footnotetext{
* Corresponding authors. Tel.: +49351463 39843.

E-mail addresses: volkan@stanfordalumni.org (H.V. Demir), alexander.eychmueller@chemie.tu-dresden.de (A. Eychmüller).
}

well as by modernization of existing technologies, which is the scope of several international programs showing already some promises and first positive results $[1,2]$. In addition to the introduction of smart energy production technologies, climate change combat may be greatly supported by the reduction and optimization of energy consumption. Nowadays sufficient and convenient lighting belongs to standard conditions of life quality in most of the developing and developed countries of the world. At the same time, lighting in these modern societies consumes up to $19 \%$ of the total energy [2]. Reduction of this number to its half would have comparable impact on the energy problem like introduction 
of wind power stations known as one of the popular alternative energy sources. For example, wind energy contributed in the year 2009 to $7.58 \%$ of the total electricity consumption in Germany [3], one of the countries with the most developed programs supporting alternative energy sources. But is it possible to increase the energy efficiency in lighting without significant decrease of life quality? One of the answers to this problem is the introduction of novel light sources replacing incandescent bulbs. Fluorescence lamps were introduced as a solution to decrease the high energy consumption of lighting [4]; however, their light quality remains very low and further decrease in the energy consumption is desired. Solid state light sources mainly known as light emitting diodes (LEDs) are among the most attractive candidates for this purpose owing to the potential of reducing the energy consumption (at least three times in comparison to incandescent light) together with exhibiting high quality lighting.

Although artificial lighting with LEDs presently attracts enormous interest [5,6], it is admitted that for the highquality white light generation mixing red, green and blue LED chip emissions is necessary. Although possible, the presently low efficiency levels of green LEDs limit the applicability of this approach [6]. Alternatively, in order to achieve pleasant and healthy lighting conditions (e.g., warm white light) by using LEDs as light sources, proper color conversion layers based on phosphors absorbing UV or blue light and re-emitting at longer wavelengths are developed. Today's traditional phosphors solely rely on the use of combinations of rare earth ions. While this conventional kind of phosphors is good at photon conversion with high quantum efficiency, there exist fundamental problems related to their photometric performance. Among them are their large emission bandwidth and inevitable emission tail towards long wavelengths, as well as difficulty in controlling the granule size, composition of layers and depositing uniform films. These disadvantages result in undesired visible color variations and complexity in tuning the color properties. Moreover, the scattering problem arises due to the large grain size causing color coordinates change over angle. On top of these scientific issues, the monopoly on the supply of the rare-earth elements emerges as a political issue potentially threatening the photonics industry, including the solid state lighting. Recently, China, currently being the main supplier of rare-earth elements has announced restrictions on the export of these materials [7]. Therefore, breaking the dependence of the photonics industry on these elements has become critical from a strategic point of view at the global level. That is why a search for novel phosphor materials exhibiting high emission quantum yields, spectral purity, long-term photo- and thermal-stability and processability (compatible integration) which do not suffer from monopoly concerns, is a key and challenging task essential to modern solid state lighting.

These aforementioned properties are generally inherent to a class of nanomaterials named semiconductor nanocrystals (NCs), alternatively also known as colloidal quantum dots (QDs). In this review we analyze and summarize the knowledge accumulated during the recent half decade on the perspectives of using semiconductor QDs in form of thin films and specially designed composite materials for the purposes of light conversion. As we assume not all our

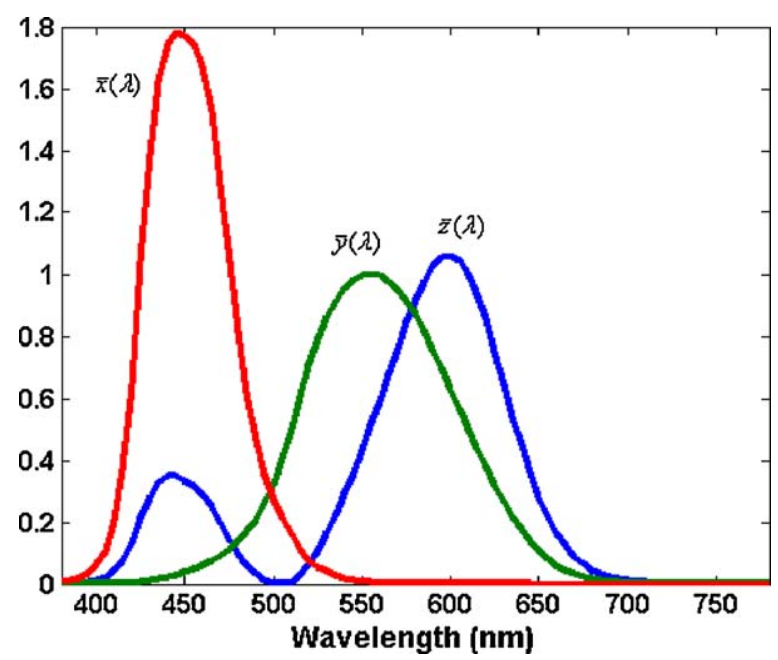

Figure 1 Spectral distribution of color matching functions [9].

readers are familiar with the terminology and standards commonly used in the field of lighting and color converters, we will begin with a general introduction into the field describing the basic parameters used to characterize the color properties of the light. Consequently, we will explain why namely quantum dots may be considered as very promising candidates for color conversion and will give examples of several concepts of utilization of QDs for these properties stressing on color mixing strategies, color design and enhancement strategies, as well as on assembly approaches necessary to integrate QDs with LED platforms. Finally we will give an outlook summarizing perspectives of the applications of the concepts overviewed.

\section{Photometric properties of white LEDs}

\section{Chromaticity coordinates}

Tristimulus coordinates $(x, y)$ represent the perceived color of a light source by the human eye. In fact, the widely reported $(x, y)$ coordinates consist of $x, y$ and $z$ coordinates. However, since the $z$ value is dependent on the $x$ and $y$ values given by $z=1-x-y$, it is not reported and instead a two dimensional chromaticity diagram of $(x, y)$ suffices. The perceived color is determined by looking at the corresponding coordinates on the standard CIE (Commission Internationale d l'Eclairage-The International Commission for Illumination) chromaticity diagram. CIE defines the international standards for color science, and the CIE 1931 color matching functions and chromaticity diagram (shown in Figs. 1 and 2, respectively) are commonly used corresponding standards in both industrial production and scientific research [5], although there are also more recent ones. However, it is also worth mentioning that the color matching functions are not unique and some other examples may be found in literature [8]. Each tristimulus value corresponds to an approximate response of each type of the red-, green-, and blue-sensitive retinal cones. Eqs. (1)-(3) are used to determine the strength of each perceived red, green and blue colors (i.e., $X, Y$ and $Z$, respectively), where $\bar{x}(\lambda), \bar{y}(\lambda)$ and 


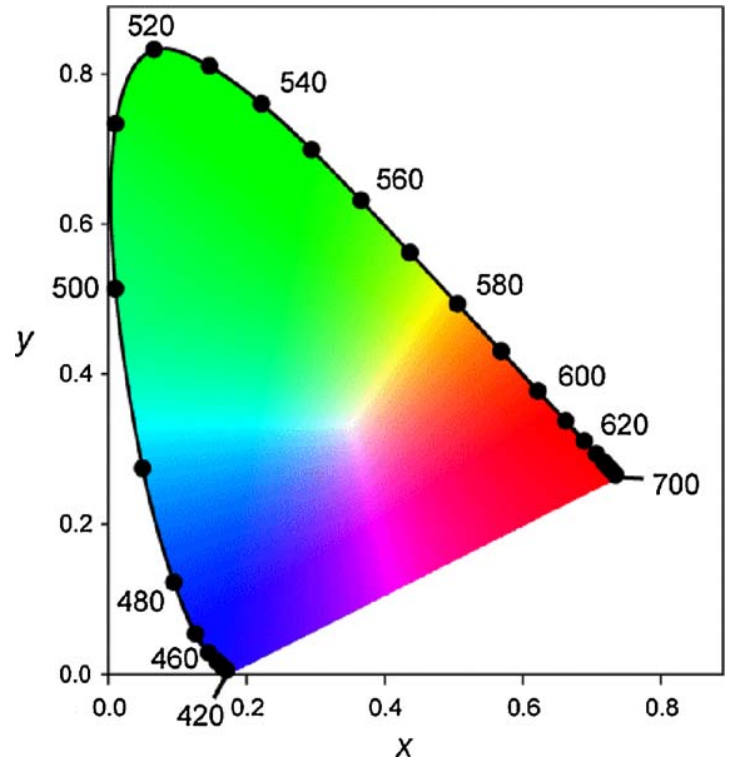

Figure 2 CIE $1931(x, y)$ chromaticity diagram [10].

$\bar{z}(\lambda)$ are the color matching functions and $P(\lambda)$ is the spectral power density.

$X=\int_{\lambda} \bar{x}(\lambda) P(\lambda) d \lambda$

$Y=\int_{\lambda} \bar{y}(\lambda) P(\lambda) d \lambda$

$Y=\int_{\lambda} \bar{z}(\lambda) P(\lambda) d \lambda$

The $(x, y)$ and $z$ chromaticity coordinates are calculated from $X, Y$, and $Z$ by normalizing them with respect to their sum using Eqs. (4) - (6). Therefore, $z$ can be extracted from $(x, y)$ as shown in Eq. (6). The equal-energy locus located in the center of the chromaticity diagram at $(x, y, z)=(1 / 3$, $1 / 3,1 / 3)$, but this might not be necessarily the desired operating point, which rather depends on the targeted application.

$x=\frac{X}{X+Y+Z}$

$y=\frac{Y}{X+Y+Z}$

$z=\frac{Z}{X+Y+Z}=1-x-y$

\section{Color temperature}

The relation between the color and temperature stems from the blackbody radiation. The $(x, y)$ tristimulus coordinates of the blackbody radiators form the Planckian locus as shown in Fig. 3 and the color temperature of a light source is determined by looking at the coordinates of the corresponding blackbody radiation temperature, whose unit is Kelvin [5]. However, in Fig. 3 the Planckian locus is only a small portion of the $(x, y)$ chromaticity diagram and there exist many operating points outside the Planckian locus. If the coordinates of a light source do not fall on the Planckian locus,

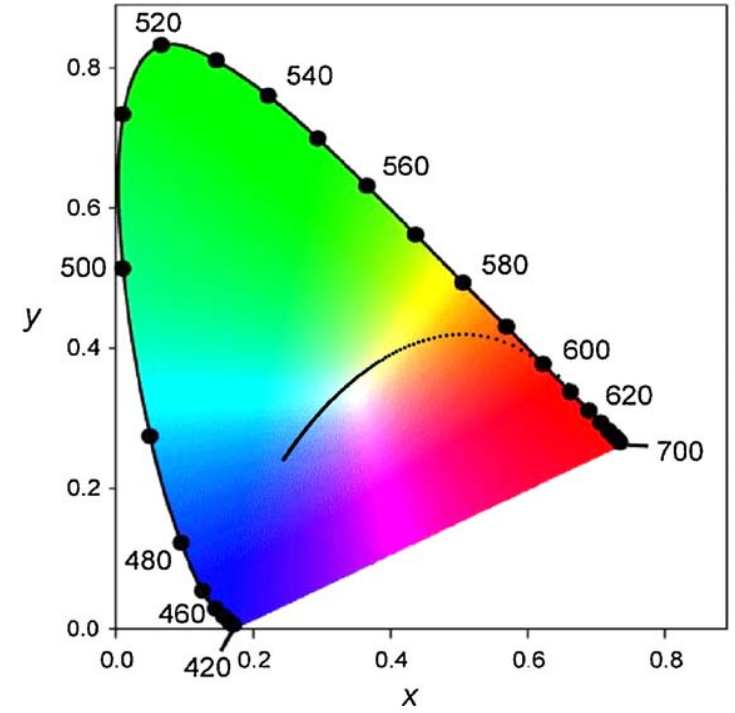

Figure 3 Planckian locus shown in the $(x, y)$ chromaticity diagram [10].

the correlated color temperature (CCT) is used to define the color temperature of a light source. CCT is calculated by transforming the $(x, y)$ coordinates of the light source to $\left(u^{\prime}, v^{\prime}\right)$ by using Eqs. (7) and (8) [11], and by determining the temperature of the closest point of the Planckian locus to the light source on the $\left(u^{\prime}, v^{\prime}\right)$ uniform chromaticity diagram (Fig. 4).

$u^{\prime}=\frac{4 x}{-2 x+12 y+3}$

$v^{\prime}=\frac{9 y}{-2 x+12 y+3}$

\section{Color rendition, color rendering index and color quality scale}

Color rendering index (CRI) is a measure of the capability of a light source to reveal the true colors of the illuminated objects. CRI ranges from -100 to 100 [12]. The best color rendering index is 100 , whereas the CRI of the poorest color rendition is -100 . A high CRI $(\geq 80)$ is required especially for indoor lighting, but for outdoor applications such as street lighting a lower CRI is sufficient for future SSL application as recommended by Sandia National Laboratories [6]. CRI of a test light source is a relative measure with respect to a reference light source, for which blackbody radiators with an excellent CRI of 100 are used in general. The incandescent lamps, which are also blackbody radiators and used for home and office illumination, have a perfect color rendering capability. However, because of their low efficiency, countries including Brazil, the European Union, Australia, Canada, and Russia phase out incandescent light bulbs for general lighting.

For the calculation of the CRI, CIE recommended 14 test color samples having unique spectral reflectivities among each other. The general CRI is calculated by using the first eight of these colors [12]. The remaining six CIE test color sample objects from 9 to 14 are used to further evaluate the CRI of the test light source. For test light sources blackbody 
Table 1 CQS and CRI of the current technologies and simulated LEDs [13-15].

\begin{tabular}{llc}
\hline Light source & $\begin{array}{l}\text { Color quality } \\
\text { scale }\end{array}$ & $\begin{array}{l}\text { Color rendering } \\
\text { index }\end{array}$ \\
\hline Incandescent & 98 & 100 \\
Mercury & 53 & 53 \\
Cool white fluorescence & 61 & 59 \\
RGB LED (470-525-630) & 55 & 31 \\
RGB LED (464-538-613) & 85 & 80 \\
RGB LED (467-548-616) & 82 & 90 \\
RGB LED (464-562-626) & 78 & 59 \\
RGB LED (457-540-605) & 74 & 80 \\
RGB LED (455-547-623) & 79 & 73 \\
\hline
\end{tabular}

radiators with the same color temperature or correlated color temperature are used depending whether the tristimulus coordinates of the test light source is on the Planckian locus or not. The color rendering index is calculated according to the test color spectral reflectivity difference under the test light source illumination and the reference light source illumination.

Although the color rendering index is widely used in the lighting community, it may have misleading results when applied to LED spectra particularly for cool white shades. Therefore, in this review we also provide information regarding a new color rendition metric called color quality scale (CQS) developed by Davis and Ohno [13-15]. The advantage of the CQS is that it provides a better scale and understanding for the color rendering capability of LED spectra while solving various problems of the CRI. Similarly, the CQS is also based on the use of a reference light source like the CRI. Different from the CRI, the CQS uses 15 reflective Munsell samples and it makes use of a saturation factor, which becomes effective when a light source enhances object chroma. In addition, the CQS ranges from 0 to 100 . The range of the CQS is also more understandable because $C R I<0$ does not express any useful information. Furthermore, the CQS value of a light source is calculated by taking the root mean square of the color differences of test samples, which are illuminated by the test and reference light sources. CQS and CRI of the current technologies and simulated LEDs are analyzed and summarized in Table 1. According to the analysis, although the CQS results are in agreement with the CRI results for traditional lamps, the CQS shows the color rendering capability of LEDs more appropriately.

\section{LER and LE}

In the lighting community, the luminous efficacy of optical radiation (LER) and luminous efficiency of the device (LE) can be mistakenly misused because the device LE is sometimes also dubbed luminous efficacy, which is different than that of optical radiation, the LER. The LER represents the relative optical power of emission spectra useful for the human eye compared to the emitted total optical power; thus, its unit is lumen per optical Watt. On the other hand, LE quantifies the same with respect to electrical power so that its unit is lumen per electrical Watt. LER and LE are

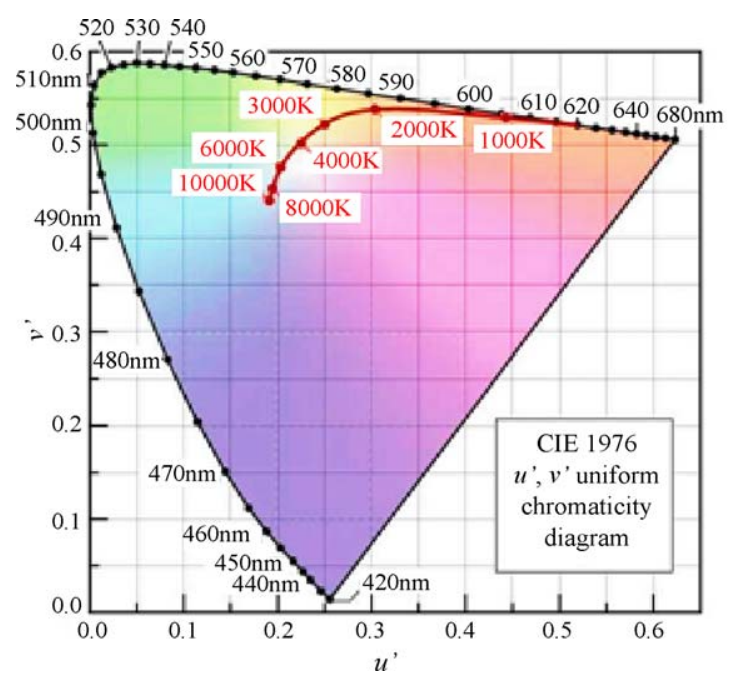

Figure 4 Chromaticity diagram $\left(u^{\prime}, v^{\prime}\right)$ along with the Planckian locus [5].

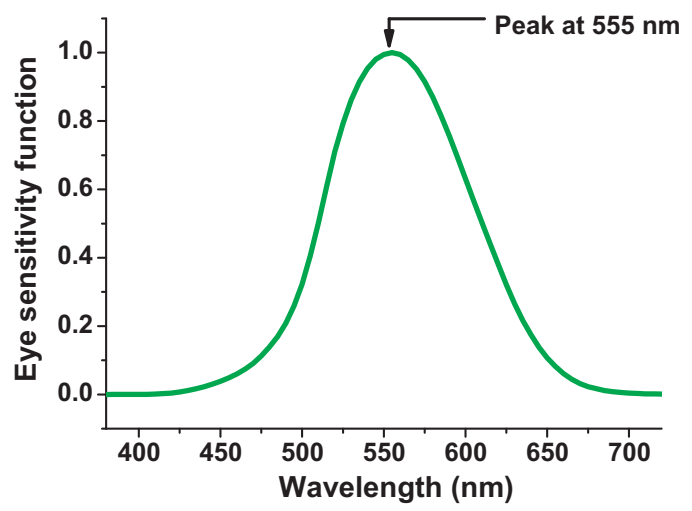

Figure 5 Eye sensitivity function spectrum (for light-adapted vision) [9].

calculated by using Eqs. (9) and (10) [16], respectively, where $v(\lambda)$ is the eye sensitivity function given in Fig. 5, $P(\lambda)$ is the spectral density (i.e., the light power emitted per unit wavelength), $I$ is the current injection level and $V$ is the applied voltage level at the operating point of the LED (with VI being the electrical input power). LE can thus be computed simply by multiplying LER by the power conversion efficiency of the LED.

$$
\begin{aligned}
& \text { LER }=\left(683 \frac{\text { Im }}{W_{\mathrm{opt}}}\right) \frac{\int P_{\mathrm{opt}}(\lambda) v(\lambda) d \lambda}{\int P_{\mathrm{opt}}(\lambda) d \lambda} \\
& \mathrm{LE}=\left(683 \frac{\mathrm{lm}}{W_{\mathrm{opt}}}\right) \frac{\int P_{\mathrm{opt}}(\lambda) v(\lambda) d \lambda}{V I}
\end{aligned}
$$

\section{Semiconductor quantum dot synthesis and optical properties required for high photometric efficiency}

Synthesis of colloidal semiconductor QDs may be divided into two main classes: those prepared in organic solvents by utilization of the hot injection approach $[17,18]$ (or its variations) and those synthesized in polar media, mainly in 
water [19-21]. Here, we are not going to describe synthetic details and make comparative analysis of the synthetic strategies, these all may be found in the recent comprehensive reviews $[18,22,23]$. The most important for our review is the fact that growing knowledge on nanocrystal syntheses and post-preparative treatments allows for almost unlimited design of the chemical composition, shape, size-distribution, surface functionalization and optical properties of the semiconductor nanocrystals [22,23]. Among the most attractive optical properties are strong, narrow, and tuneable (quantum confinement) photoluminescence with quantum yields in the range from 30 - to over $90 \%$ and full-width at half-maxima (FWHM) of $20-50 \mathrm{~nm}$ in the visible spectral region. Moreover, other important and attractive properties of the nanocrystal QDs are their high extinction coefficients (at least an order of magnitude higher than organic dyes) and their stability (common for inorganic materials). Furthermore, the existence of functional molecules (so-called ligands or stabilizers) on the QD surface which may be introduced during the synthesis or exchanged post-preparatively enables researchers to design their chemical compatibility with various solvents and coupling with other nanoparticles, surfaces and chemical substances by means of electrostatic or covalent bonding [24]. The combination of all these favorable properties makes QDs promising candidates for a variety of active applications in nanophotonics including color-conversion LEDs [23].

At the first glance it would seem that a material with a narrow emission spectrum is not the best candidate for white light generation. However, if one takes into account the variety of the emitters available (including those of the same composition and surface functionality, but only different in size) and the ability of the researches to combine them in composite structures and blends, the advantage of the QDs for precise color design becomes much more obvious [25]. Recently a photometric study of ultra-efficient light emitting diodes is reported exhibiting advanced CRI and LER using semiconductor QDs luminophores. Over 200 million systematically varied QD-LED designs have been simulated to understand a feasible performance in terms of CRI vs. LER and the results are demonstrated in Fig. 6 [26]. According to the results, warm-white LEDs with CRI $>90$ and LER $>380 \mathrm{~lm} / \mathrm{W}_{\text {opt }}$ at a correlated color temperature of $3000 \mathrm{~K}$ are achievable via the proper spectral engineering of $Q D$ luminophores.
To achieve high performance, the selection of narrow emitters is of great importance. For numerical analyses, we investigated four color mixing designs to generate photometrically high-quality white light [26]. In this study, we found out that only ca. $0.001 \%$ of over 200 million designs can actually meet the high performance criteria of CRI $>90$ and LER $>380 \mathrm{~lm} / W_{\text {opt }}$ in the warm white region. Further investigation of their average and standard deviation values that overcome these thresholds showed that the red wavelength should be strategically selected around $621 \mathrm{~nm}$ while wavelengths of other color components should be placed around 465, 528, and $569 \mathrm{~nm}$ for blue, green, and yellow, respectively. Except for the red component, the standard deviation of all colors is about $10 \mathrm{~nm}$, which allows for a relatively large room to tune the peak emission wavelength. However, the optimal red wavelength is rather restricted; the red emitter should not deviate from its targeted average value as its standard deviation is only $2 \mathrm{~nm}$. Looking at FWHMs of all the four-color components, we again discovered a striking difference between the red and other colors. For blue, green, and yellow, the FWHM should be around $44 \mathrm{~nm}$, together with a standard deviation of ca. $8 \mathrm{~nm}$, which leaves large enough room to tolerate relatively broad linewidths. However, the red FWHM should be significantly narrower, with its average FWHM of $32 \mathrm{~nm}$ and standard deviation of $3.5 \mathrm{~nm}$. Finally, the investigation of average relative photon counts of every color component revealed that the blue needs to be weak in the spectrum (79.3 units out of 1000) whereas the red should be very dominant (469.3 units out of 1000). Meanwhile, the green and yellow may take intermediate values (211.4 and 240.1 units out of 1000 , respectively). Their standard deviation value further pointed out that the exact weights of the blue and red (and thus their balance) are very critical while the end photometric performance is less sensitive to the exact contributions coming from the green and yellow components as long as they are reasonably in the mid-range. Fig. 7 shows a LED spectrum generated using quantum dot nanophophors with these stated average values, given along with the resulting set of photometric figure-of-merits of $\mathrm{CRI}=91.3$, LER $=386 \mathrm{~lm} / \mathrm{W}_{\mathrm{opt}}$, and CCT $=3041 \mathrm{~K}$ in the inset. This shows that it is in principle possible to obtain spectrally efficient, high-quality white light by using quantum dots.

In addition to these parametric analyses, we investigated the fundamental relations and trade-offs between the photometric performance criteria. One interesting outcome (a)

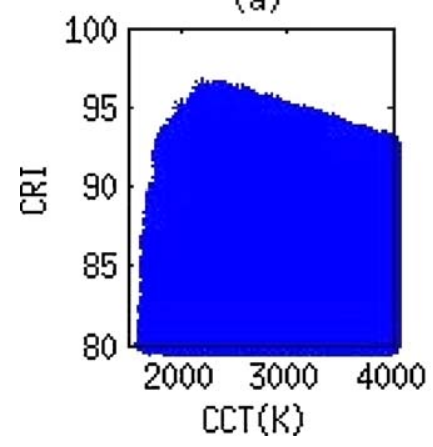

(b)

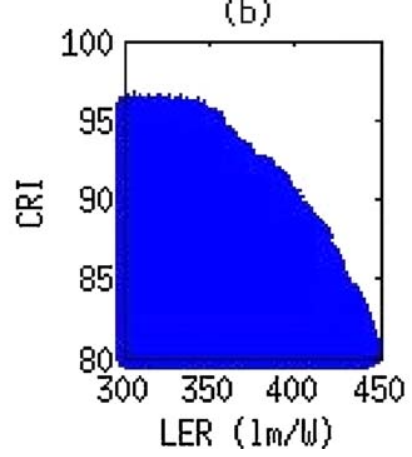

(c)

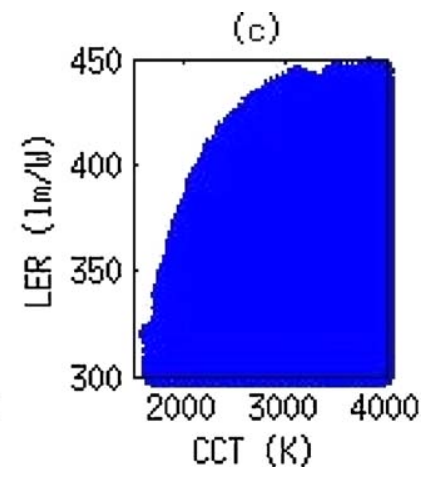

Figure 6 Relations between (a) CRI and CCT, (b) CRI and LER, and (c) LER and CCT [26]. 


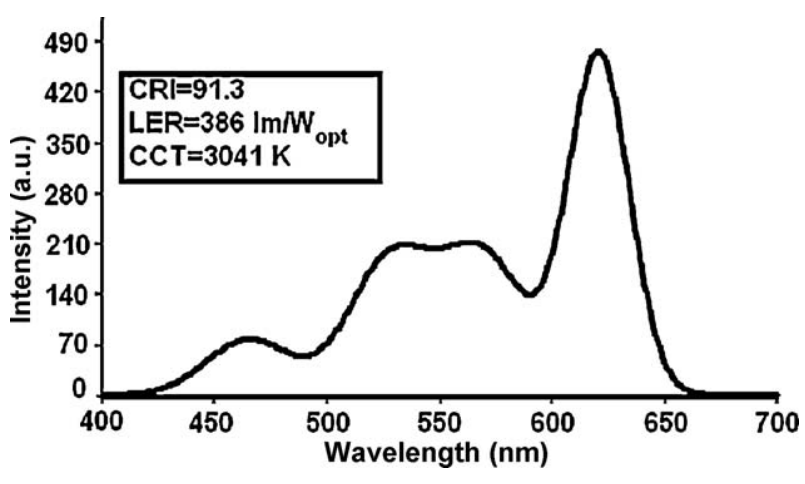

Figure 7 Exemplary spectrum generated using quantum dot nanophosphors (with the stated average values of the numerical analyses), together with the resulting photometric performance [26].

of this study was to find that the high CRI comes at the expense of significantly reduced LER, as depicted at different CCT values (with ever increasing cost at a lower CCT) in Fig. 8. An increased CRI requires decreasing the CCT. But, cooler white light sources (i.e., higher CCTs) are needed for increased LER values. Therefore, there is an intrinsic trade-off pairwise between the LER and the CRI, and the LER and the CCT when making a spectrally efficient, high rendering, warm white LED. This effort requires careful optimization.

\section{Nanocrystal quantum dot-based photonic-color-conversion LEDs}

The operation of color conversion white LEDs is based on the mutual use of LEDs and integrated color converting films. When the LED is turned on it generates electroluminescence and its electroluminescence pumps the integrated color conversion layer, which in turn generates photoluminescence. Consequently, the electroluminescence of the LED and the photoluminescence of the color conversion layer collectively generate white light.

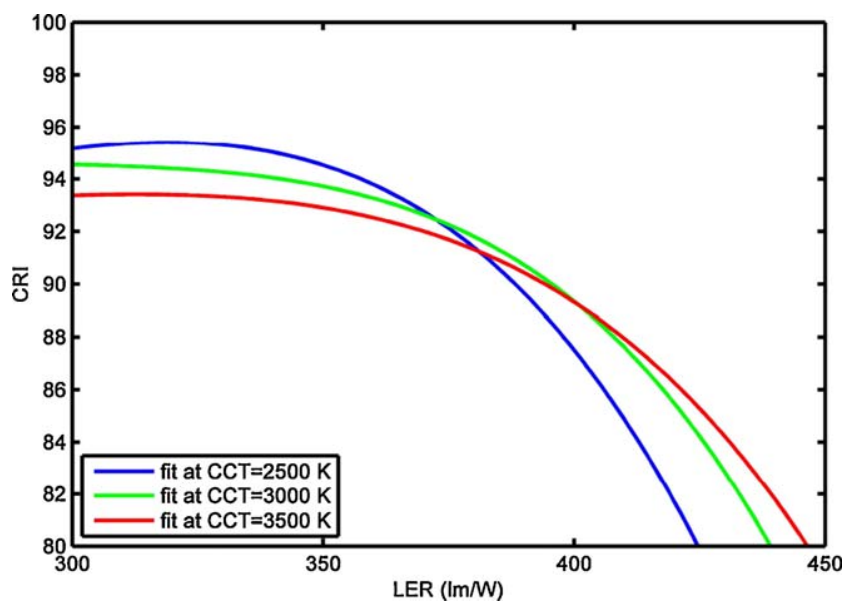

Figure 8 Feasibility of CRI vs. LER at different CCTs using quantum dot nanophosphors.

\section{Multilayered QD integrated LEDs}

The use of combinations of QDs at different emission colors enables color emission [27], which one can utilize to make a convenient adjustment and optimization of the white light parameters, as reviewed here. To fine-tune the generated white light spectrum, the size and concentration of the QDs and the thickness and order of the QD films are needed to be carefully adjusted. The size of the QDs determines the colors that will contribute to white light generation. The QD concentration and the QD film thickness affect the level of the photoluminescence by the color conversion layer and the transmitted electroluminescence by the LED. Furthermore, the integration order of differently sized QD layers affects the reabsorption of radiation by the previous QD layers. Therefore, the capability to set properly such device parameters allows us to obtain the aimed white light spectrum having high photometric quality [28].

By using this approach Nizamoglu et al. demonstrated tuneable white light generation utilizing combinations of cyan-, green-, yellow- and red-emitting $\mathrm{CdSe} / \mathrm{ZnS}$ core-shell nanocrystals on a blue-LED [28]. Four different white LEDs are fabricated that include combinations of (1) yellow-emitting nanocrystals $\left(\lambda_{\mathrm{PL}}=580 \mathrm{~nm}\right)$ on a blue LED $\left(\lambda_{\mathrm{EL}}=440 \mathrm{~nm}\right)$ with $(x, y)=(0.37,0.25), \mathrm{CCT}=2692 \mathrm{~K}$ and $C R I=14.69$; $(2)$ cyan- and red-emitting nanocrystals $\left(\lambda_{\mathrm{PL}}=500\right.$ and $\left.620 \mathrm{~nm}\right)$ on a blue LED $\left(\lambda_{\mathrm{EL}}=440 \mathrm{~nm}\right)$ with $(x$, $y)=(0.37,0.28), C C T=3246 \mathrm{~K}$ and $\mathrm{CRI}=19.65$; (3) green-, yellow- and red-emitting nanocrystals $\left(\lambda_{\mathrm{PL}}=540,580\right.$ and $620 \mathrm{~nm})$ on a blue LED $\left(\lambda_{\mathrm{EL}}=452 \mathrm{~nm}\right)$ with $(x, y)=(0.30$, $0.28), C C T=7521 \mathrm{~K}$ and $\mathrm{CRI}=40.95$; and (4) cyan, green, yellow and red nanocrystals $\left(\lambda_{\mathrm{PL}}=500,540,580\right.$ and $\left.620 \mathrm{~nm}\right)$ on a blue LED $\left(\lambda_{E L}=452 \mathrm{~nm}\right)$ with $(x, y)=(0.24,0.33)$, $C C T=11,171 \mathrm{~K}$ and $\mathrm{CRI}=71.07$ shown in Fig. 9. Chen et al. have achieved a better CRI by using QD luminophores. The authors combined blue InGaN chips with red- and green-emitting colloidal core/shell CdSe/ZnSe QDs with the photoluminescence $(\mathrm{PL})$ quantum efficiency $(\mathrm{QE})$ exceeding $40 \%$. The fabricated white LED exhibits a CRI of 91 at the equal-energy point with tristimulus coordinates of $(0.33$, 0.33) [29].

Tuneable white light generation with combinations of QDs allows the QD integrated LEDs to be used in any specific application via proper spectral engineering. In one of the recent papers, QD-LEDs are prepared for indoor lighting, which is one of the most important lighting applications because of the demand for warm white light sources with high color rendering indices [25]. To meet these needs, QD hybridized warm-white LEDs are promising candidates to achieve CCTs below $4000 \mathrm{~K}$ for warm-white light generation and a CRI above 80 for future solid state lighting applications [30]. To generate such a high quality white light, greenand red-emitting CdSe/ZnS core-shell QDs emitting at 555 and $613 \mathrm{~nm}$ are hybridized on a blue InGaN/GaN LED emitting at $452 \mathrm{~nm}$. The advantage of using QD luminophores is the elimination of far red emission $(>650 \mathrm{~nm})$ because of their narrow linewidths. As a result, high LER values become achievable. However, when conventional phosphors are used for color conversion, a strong emission tail beyond $650 \mathrm{~nm}$ is observed, which significantly decreases the LER. Thus, optically efficient white light generation necessitates the use of QDs instead of conventional phosphors. 

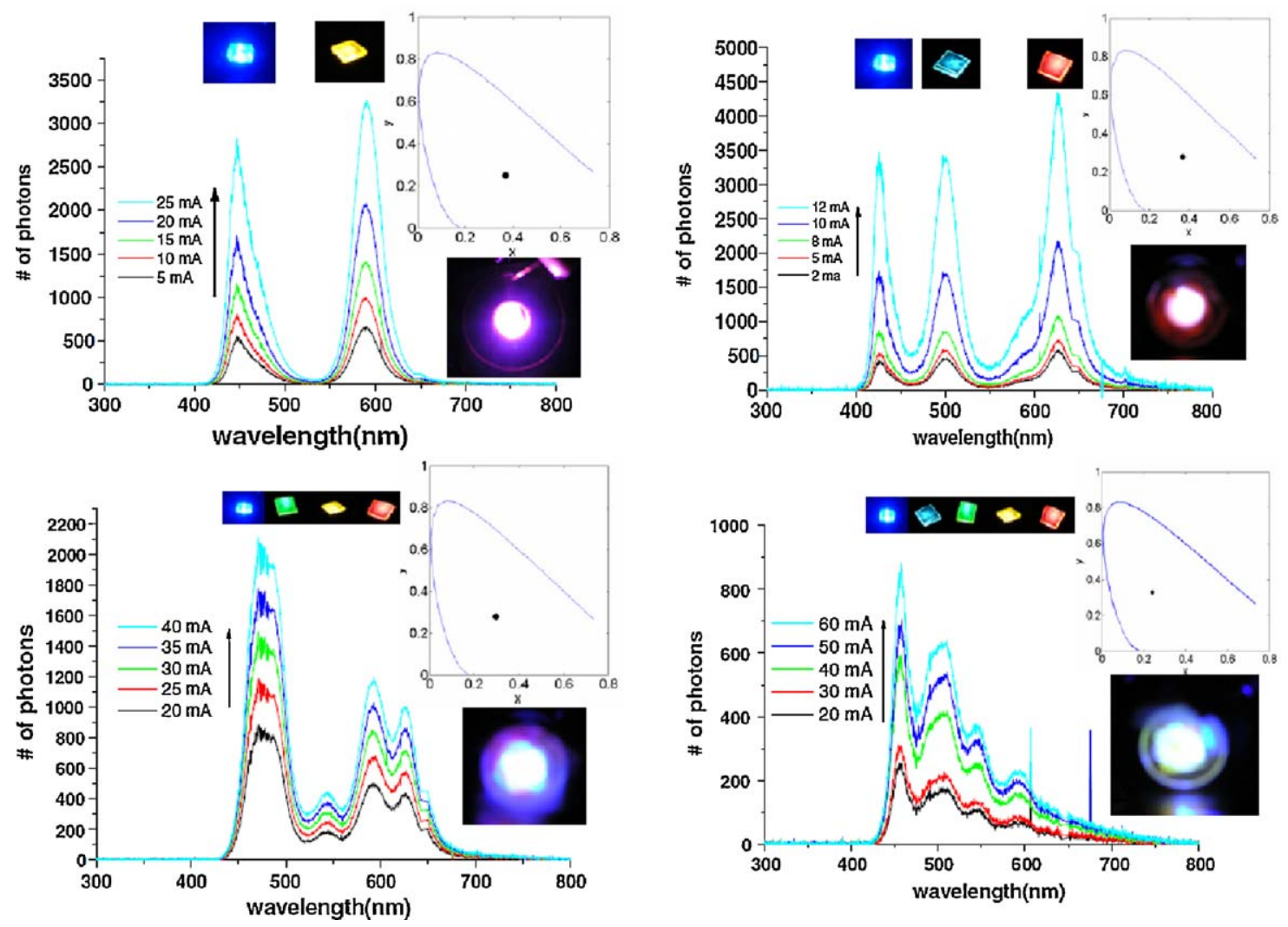

Figure 9 Luminescence spectra of (a) yellow-emitting QDs integrated on a blue LED at $440 \mathrm{~nm}$, (b) cyan- and red-emitting QDs on the blue LED at $440 \mathrm{~nm}$, (c) green-, yellow- and red-emitting QDs on the blue LED at $452 \mathrm{~nm}$ and (d) green-, cyan-, yellow- and red-emitting QDs on the blue LED at $452 \mathrm{~nm}$ at increasing current levels, and $(x, y)$ coordinates of the hybrid white LED and pictures of the resulting hybrid white LED and used QDs [28].

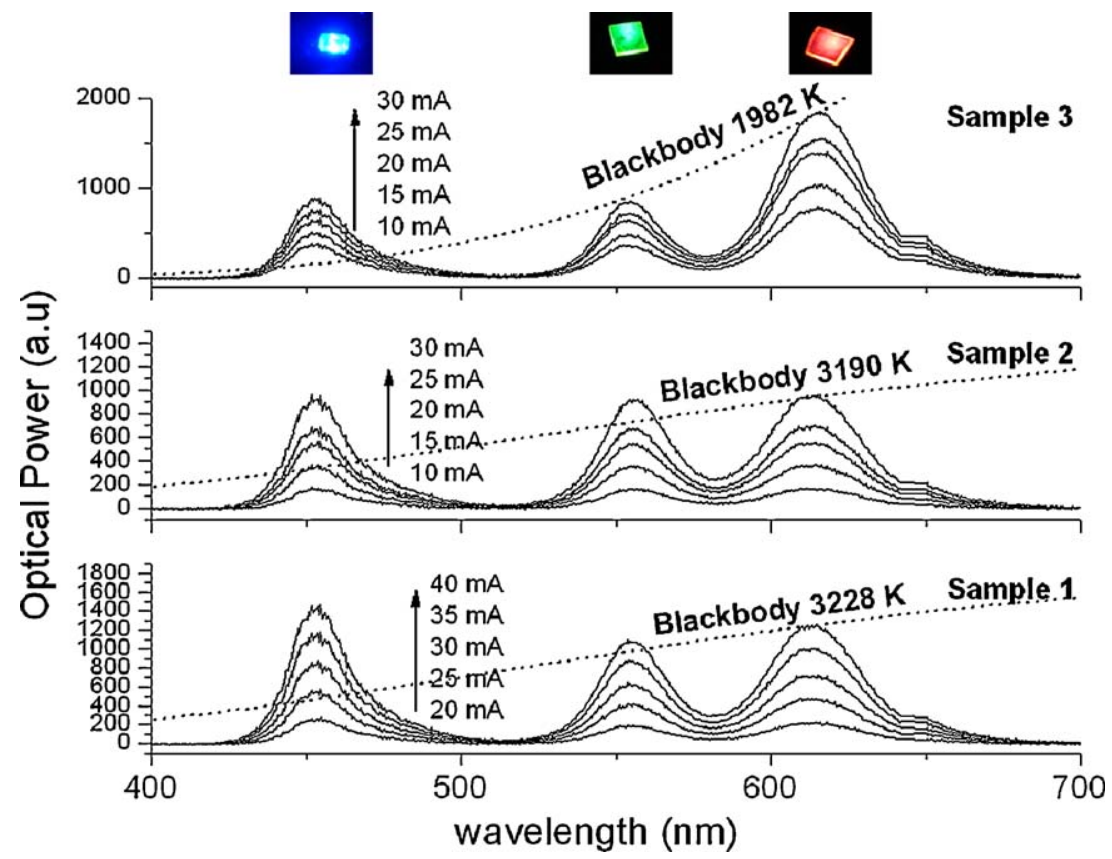

Figure 10 Luminescence spectra of Samples 1-3 at different current injection levels. Reprinted with permission from Reference [25]. Copyright 2008 American Institute of Physics. 
Furthermore, to obtain high quality white light, blackbody radiators with low CCTs are explored and their spectra are mimicked using QDs because they have a natural CRI of 100 with a warm white appearance. In Fig. 10 the blackbody radiation at the color temperature of the implemented hybrid warm-white LEDs (Samples 1-3) are illustrated. To generate warm white light, the red content is required to be raised, while keeping the spectrum within the white region. The luminescence of the first QDLED corresponds to $(x, y)=(0.37,0.30)$, LER $=307 \mathrm{~lm} / W_{\text {opt }}$, $C R I=82.4$, and $C C T=3228 \mathrm{~K}$. This CCT value brings the generated color in the warm-white region and also its CRI is higher than 80 , which fulfills the requirement for future lighting applications $[3,13,26]$. Furthermore, the LER rises above $300 \mathrm{~lm} / W_{\text {opt }}$, which is a significantly high optical efficiency for warm-white LEDs. The second QD-LED is generated by increasing the ratio of the red-emitting QDs. The corresponding emission spectrum leads to $(x, y)=(0.38$, $0.31)$, LER $=323 \mathrm{~lm} / \mathrm{W}_{\mathrm{opt}}, \mathrm{CRI}=81.0$, and CCT $=3190 \mathrm{~K}$. Since the integrated number of red-emitting QDs is increased, the emission turns into a warmer white shade with a lower CCT while keeping the LER above $300 \mathrm{~lm} / W_{\text {opt }}$. Further increase of the red-content results in $(x, y)=(0.37,0.30)$, $\mathrm{LER}=303 \mathrm{~lm} / \mathrm{W}_{\text {opt }}, \mathrm{CRI}=79.6$, and CCT $=1982 \mathrm{~K}$. In this case, the $(x, y)$ coordinates reach almost the edge of the white region, as shown in Fig. 11, meaning that this operating point is one of warmest white light points.

Another important application of QD-LEDs is the display backlighting, which makes use of their spectral tuneability. Samsung Advanced Institute of Technology reported on the synthesis of QDs with high QE, hybridization of these efficient emitters on a blue LED and integration of white LEDs into a 46-inch LCD TV backlighting unit [31]. For these green- and red-emitting QDs CdSe//ZnS/CdSZnS and $\mathrm{CdSe} / \mathrm{CdS} / \mathrm{ZnS} / \mathrm{CdSZnS}$ are synthesized with their reported in-solution QE of $100 \%$ and $95 \%$, respectively (where // means alloy between two materials). The high QE of these QD emitters also prove a significant reduction of surface states and their blinking free behavior. After integrating these green- and red-emitting QDs (with optical densities of 0.035 and 0.0035 ) on a blue LED in the silicone encapsulant,

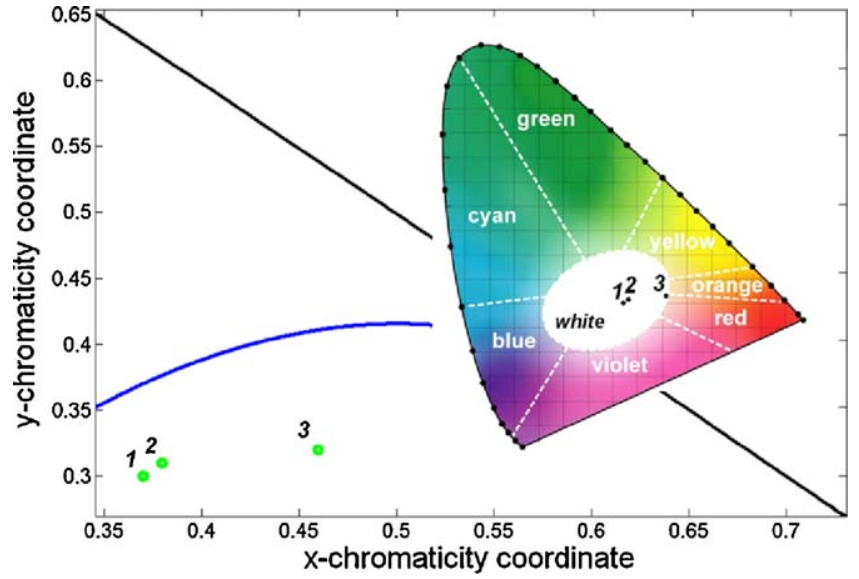

Figure 11 Zoomed $(x, y)$ tristimulus coordinates of Samples $1-3$. Blue curve denotes the Planckian locus (chromaticity points of the blackbody radiator) whereas the black line is the border of the $(x, y)$ chromaticity diagram. The inset shows the $(x, y)$ tristimulus coordinates on the CIE chromaticity diagram. Reprinted with permission from Reference [25]. Copyright 2008 American Institute of Physics.

their QEs dropped to $59 \%$ and 39\%, respectively. This efficiency drop can be explained by the introduction of defect centers because of broken ligands or interaction with encapsulants at high temperatures above $150^{\circ} \mathrm{C}$. Furthermore, reabsorption and scattering effects due to aggregation of QDs may also contribute to this efficiency drop. The resultant spectra of the white LED are given in Fig. 12a having $(x, y)$ tristimulus coordinates of $(0.24,0.21)$ with a CCT of $100,000 \mathrm{~K}$ and LE of $41 \mathrm{~lm} / \mathrm{W}_{\text {elect }}$. For the purposes of comparison, a blue LED is hybridized with an Eu-doped $\beta$-SiAION green phosphor and an Eu-doped $\mathrm{CaAlSiN}_{3}$ red phosphor. This phosphor based white LED has an LE of $40 \mathrm{~lm} / W_{\text {elect }}$. Although the radiation power of the phosphor is better than that of the QDs, a considerable amount of the radiation from the blue LED and the red phosphor could not contribute to the LER (because of being on the lower sides of the eye sensitivity function). As a result, the LE remains significantly
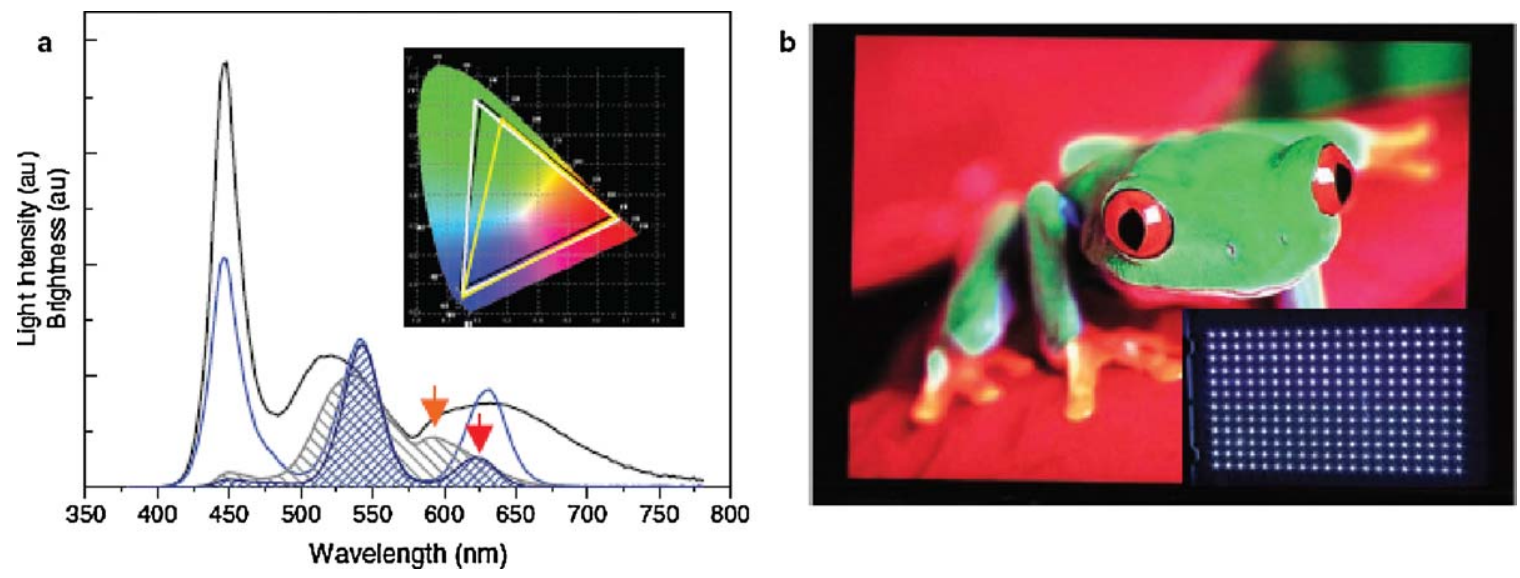

Figure 12 (a) Light intensity spectra (solid line) and brightness (hatched area) of the QD-LED (blue) and the phosphor-LED (grey). Inset: Color triangles of the QD-LED (white) and the phosphor-LED (yellow) compared to NTSC1931 (black). (b) A display image of a 46 inch LCD TV panel and a quarter of the white QD-LED backlights (inset). Reprinted with permission from Reference [31]. Copyright 2010 WILEY-VCH Verlag GmbH \& Co. KgaA, Weinham. 
low. In addition to this advantage of QD-LEDs over phosphor LEDs, the QD-LEDs define a wider color gamut. The area of the RGB triangle of the white QD-LED exceeds the area of the National Television System Committee (NTSC) color space (104.3\%) whereas the phosphor-LED can cover only $85.6 \%$, meaning that a better color quality can be obtained by using QD-LEDs. The display image of a 46-inch LCD TV panel and a quarter of the white QD-LED backlights are shown in Fig. 12b.

An important question that should be answered about light sources is the lifetime because it has a direct effect on the cost of the lighting systems. It has been shown by Jang et al. that the half-lifetime of the silicone encapsulated QD-LEDs is beyond $15,000 \mathrm{~h}$, which is comparable to the typical lifetimes guaranteed by mobile display systems [31]. Another finding of this work has been that the emission peaks did not change upon an excitation for $2200 \mathrm{~h}$, which is also important to preserve the designed spectrum.

\section{Dual-color emitting QD integrated LEDs}

Dual-color emitting nanostructures based on quantum wells (QWs) are investigated as another white light generation source. The main aim of this approach is to obtain a controllable broad band emission from individual QDs so that white light emission with high photometric quality can be generated. As an interesting example, Chen et al. fabricated a blue/green two-color emitting LED based on the mixture of two kinds of epitaxially grown QWs. The redemitting QDs were further integrated on top of this LED for white light generation. However, this LED exhibits different color properties at various current injection levels because of the electron and hole injection imbalance. At relatively low (high) current injection levels, the electroluminescence of green-emitting (blue-emitting) QW dominates [32]. As a possible solution to the color stability issue, Nizamoglu et al. hybridized dual-color emitting QD-QW CdSe/ZnS/CdSe multi-layered heteronanocrystals on InGaN/GaN LEDs for high-quality white light generation [33]. Generally, white LEDs are achieved by using combinations of single color emitting QDs, but novel dual-color emitting QD-QW heteronanocrystals also offer advantages in terms of white light generation via integration of a single type of QDs only. These heteronanocrystals are composed of a CdSe QD core surrounded by a ZnS shell and then a CdSe shell again. Sapra et al. have previously showed white light generation using these dual (cyan and red) QD emitters in toluene solution [34]. However, the CRI of the generated spectrum was low and these white emitters were not integrated into devices. For the proper white light generation, specially designed dual color (green and red) emitting QDs were fabricated and integrated on a blue LED. The emission spectra and true color image of the operating LED are provided in Fig. 13 [33]. These spectra correspond to chromaticity coordinates of $(x, y)=(0.36,0.30)$, and have a photometric performance of $L E R=278 \mathrm{~lm} / W_{\text {opt }}, C C T=3929 \mathrm{~K}$, and CRI $=75.1$. The main reason of this lower photometric performance compared to the integration of $\mathrm{QD}$ multilayers is the difficulty in the control of emission spectra of dual-color emitting QDs.

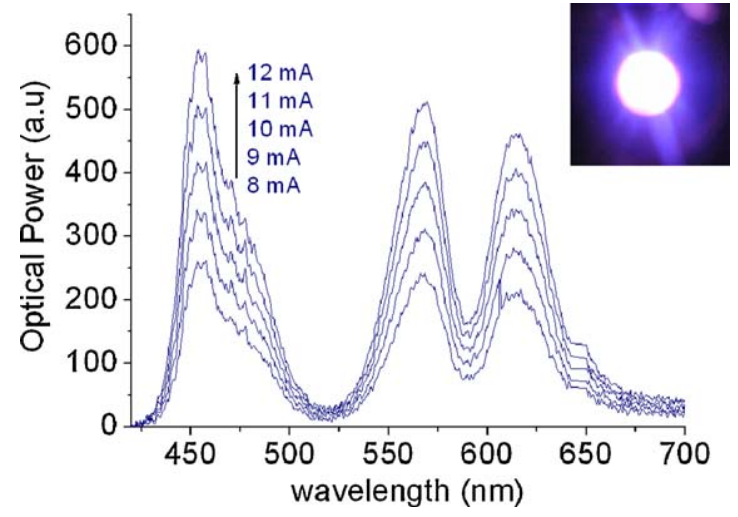

Figure 13 Luminescence spectra of onion-like $\mathrm{CdSe} / \mathrm{ZnS} / \mathrm{CdSe}$ core/shell/shell heteronanocrystals hybridized on a blue light emitting diode driven at different levels of current injection at room temperature, along with a picture of the resulting hybrid QD-white LED while generating white light. Reprinted with permission from Reference [33]. Copyright 2008 American Institute of Physics.

\section{Trap-rich QD integrated LEDs}

Trap-rich QDs are also employed for white light generation. The emission from trap states being due to surface defects covers the entire visible spectrum. When these QDs are pumped, white light generation is achieved via recombination through the interband and mid-gap states. High quality white light generation in the above mentioned examples is generally achieved with the combined luminescence from QDs emitting at different colors on a blue LED, for which also the transmitted intensity coming from the blue LED needs to be properly adjusted. As a conceptual progress, integration of white emitting QDs on near-UV LEDs provides an easier device fabrication and engineering. To generate and tune white light, CdS QDs were integrated by drop-casting and the $(x, y)$ tristimulus coordinates were properly tuned to warmer CCT with an increased number of hybridized QDs as shown in Fig. 14 [35]. The last sample leads to the optical properties of $(x, y)=(0.35,0.40), C C T=4718 \mathrm{~K}$ and $C R I=74.9$. In another study, Gosnell et al. deposited CdSe QDs on a nearUV LED and the optical properties of white light for various QD layer thicknesses were investigated as summarized in Table 2 [36]. High-quality white LEDs with a CRI even higher than 90 were acquired using these QDs, but the LE appeared to be less than $5.3 \mathrm{~lm} / W_{\text {elect }}$ meaning that the power conversion efficiencies are less than $2 \%$. Although these QDs allow for easy fabrication and engineering, their efficiencies are limited.

\section{Co-integration of QDs, polymers and phosphors on LEDs}

Another color-conversion LED method is the blend of QDs and other light-emitting materials (e.g., polymers) [36,37]. In a recent study blue-emitting 9,9-bis(2ethylhexyl)polyflourene is used with green-, yellow- and red-emitting $\mathrm{CdSe} / \mathrm{ZnS}$ core/shell QDs together [38]. Since QDs suffer from low efficiency in the blue region of the spectrum, relatively more efficient blue-emitting polyflourene 
Table 2 Luminous flux (in lumens) of nanocrystal-polymer films excited by near-UV LEDs emitting at the wavelengths of 365, 385 , and $405 \mathrm{~nm}$ for various film thicknesses divided by the output power of the UV-LED alone (in W), as well as the corresponding CIE chromaticity coordinates and CRI. A symbol of "_." ' in the CRI column is used to indicate that the spectrum is too imbalanced to be considered as white light [36].

\begin{tabular}{|c|c|c|c|c|c|c|c|c|c|}
\hline \multirow[t]{2}{*}{ Thickness $(\mu \mathrm{m})$} & \multicolumn{3}{|l|}{$365 \mathrm{~nm}$} & \multicolumn{3}{|c|}{$385 \mathrm{~nm}$} & \multicolumn{3}{|c|}{$405 \mathrm{~nm}$} \\
\hline & $\mathrm{Im} / W$ & $(x, y)$ & CRI & $\mathrm{lm} / W$ & $(x, y)$ & CRI & $\operatorname{lm} / W$ & $(x, y)$ & CRI \\
\hline 6 & 2.7 & $0.24,0.17$ & - & 3.1 & $0.21,0.09$ & - & 2.2 & $0.18,0.02$ & - \\
\hline 21 & 4.5 & $0.28,0.26$ & 88 & 4.1 & $0.23,0.14$ & - & 4.2 & $0.19,0.06$ & - \\
\hline 61 & 4.9 & $0.35,0.37$ & 86 & 5.1 & $0.31,0.28$ & 82 & 3.8 & $0.23,0.13$ & - \\
\hline 143 & 5.0 & $0.40,0.42$ & 84 & 5.3 & $0.38,0.38$ & 82 & 3.1 & $0.31,0.26$ & 74 \\
\hline 220 & 4.5 & $0.41,0.42$ & 88 & 4.7 & $0.39,0.38$ & 87 & 3.1 & $0.35,0.33$ & 90 \\
\hline 418 & 4.7 & $0.44,0.43$ & 88 & 4.5 & $0.41,0.39$ & 86 & 2.6 & $0.37,0.34$ & 88 \\
\hline 1212 & 3.9 & $0.46,0.45$ & 86 & 4.1 & $0.42,0.39$ & 84 & 2.5 & $0.38,0.34$ & 86 \\
\hline
\end{tabular}

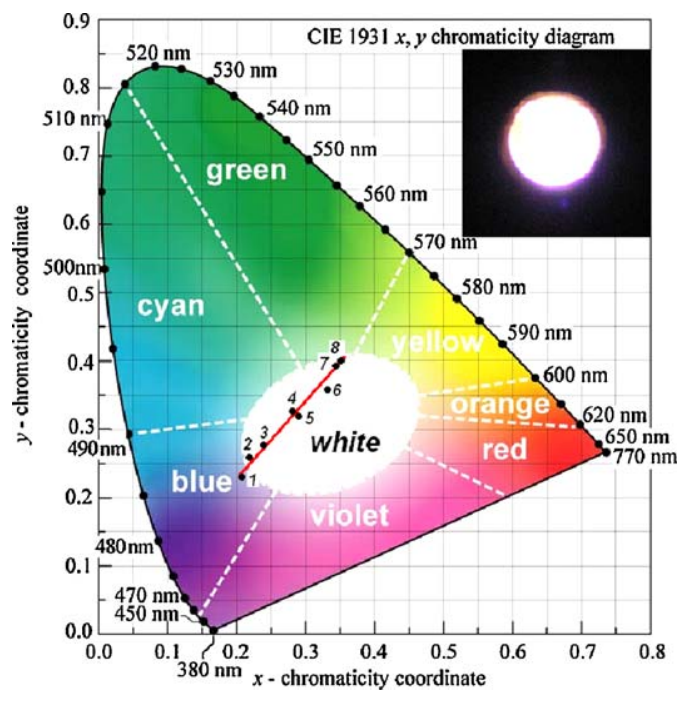

Figure $14(x, y)$ chromaticity diagram of white emitting CdS nanoluminophores tuned with their amount hybridized on the LED [35].

is a perfect candidate to achieve high-quality white light. By using this combination, white light generation with a CRI of 83.0 is acquired. In another study, eminently greenish-yellow-emitting $\mathrm{Sr}_{3} \mathrm{SiO}_{5}: \mathrm{Ce}^{3+}, \mathrm{Li}^{+}$and high quality TOP/TOPO/HDA-capped CdSe QDs are integrated on a blue LED $[39,40]$. The white LEDs exhibited an LE of $14.0 \mathrm{~lm} / \mathrm{W}_{\text {elect }}$, a CCT of $8864 \mathrm{~K}$ and an excellent CRI of 90.1 [39]. Jang et al. demonstrated a bright three-band white LED which is based on $\mathrm{CdSe} / \mathrm{ZnSe}$ quantum dots and $\mathrm{Sr}_{3} \mathrm{SiO}_{5}: \mathrm{Ce}^{3+}$, $\mathrm{Li}^{+}$phosphors, as shown in Fig. 15 [39]. The hybrid white LED performance corresponds to a LE of $26.8 \mathrm{~lm} / W_{\text {elect }}$, a CCT of $6140 \mathrm{~K}$, and a CRI of 85 . Also, as an example of application of Cd-free QDs for white light conversion properties Ziegler et al. showed a white LED with a CRI of 86 by coating the blend of red-emitting silica-coated InP/ZnS nanocrystals with green-emitting $\mathrm{Sr}_{0.94} \mathrm{Al}_{2} \mathrm{O}_{4}: \mathrm{Eu}_{0.06}$ converters and yellow $\mathrm{Y}_{2.94} \mathrm{Al}_{5} \mathrm{O}_{12} \mathrm{Ce}_{0.06}$ on a blue LED [41].

\section{FRET-converted LEDs}

In this part, we focus on the utilization of Förster-type resonance energy transfer (FRET) from quantum wells (QWs) to QDs for white light generation. Since the excitons generated in the QWs can be directly, efficiently and nonradiatively transferred to QDs via FRET, a lower number of integrated particles are needed for color conversion. Furthermore, the $\mathrm{QE}$ of the hybrid device does not directly depend on the external QE of the QWs. Indeed, as opposed to electroluminescence, the excitons of QWs pump the QDs via FRET directly, i.e., without recombining in the QWs. Utilization of FRET requires short distances between the donor and acceptor which means that thin QD layers are needed. This
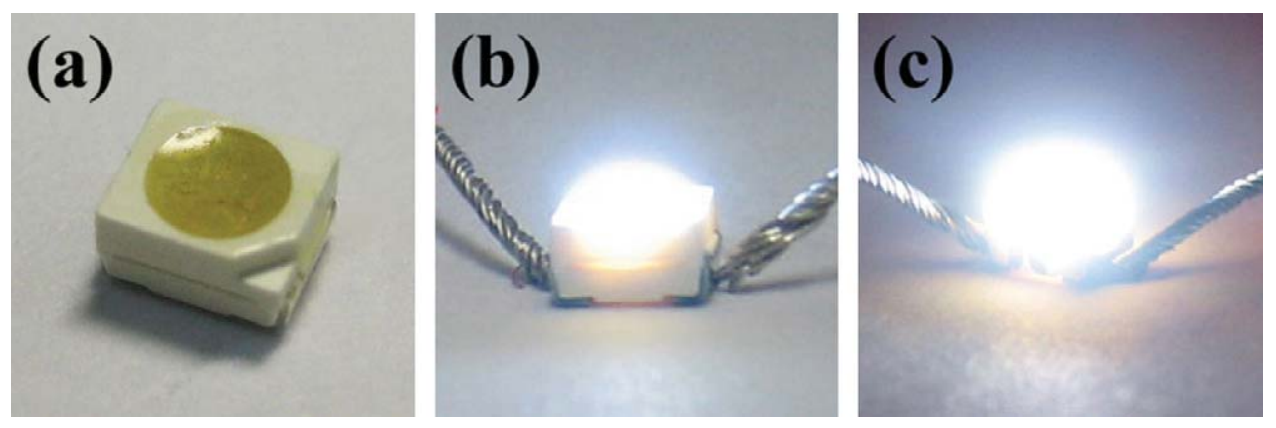

Figure 15 Photographs of (a) an as-prepared $\mathrm{CdSe} Q \mathrm{QD}$ - and $\mathrm{Sr}_{3} \mathrm{SiO}_{5}: \mathrm{Ce}^{3+}, \mathrm{Li}^{+}$phosphor-based white LED, (b and c) the same white light-emitting LED operated at $5 \mathrm{~mA}$ and $20 \mathrm{~mA}$, respectively. Reprinted with permission from Reference [39]. Copyright 2008 WILEY-VCH Verlag GmbH \& Co. KgaA, Weinham. 


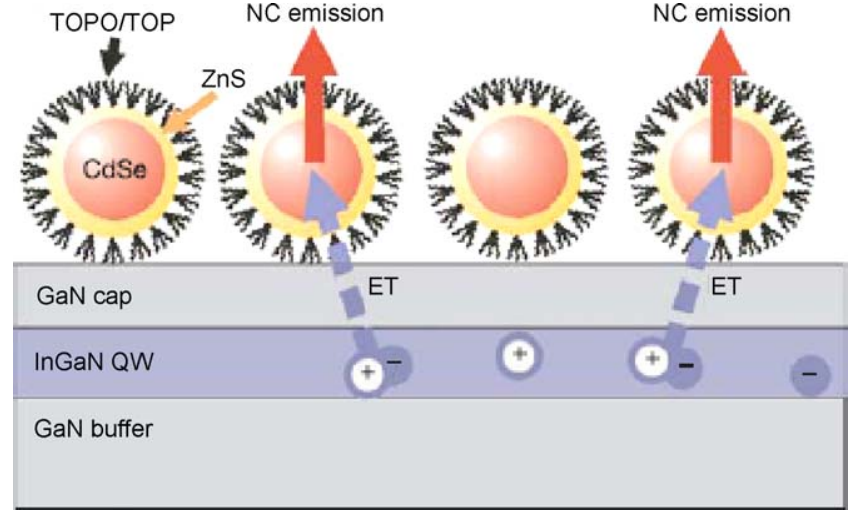

Figure 16 Schematic of the hybrid quantum-well/nanocrystal structure. The structure consists of an InGaN/GaN quantumwell heterostructure with a monolayer of TOPO/TOP-capped $\mathrm{CdSe} / \mathrm{ZnS}$ core/shell nanocrystals on top of it. Electron-hole pairs generated in the quantum well can experience nonradiative energy transfer into the QDs by Förster-type, dipole-dipole interactions. The emission properties of the QDs excited by FRET are determined by the QD size. Reprinted by permission from MacMillan Publishers Ltd: Nature [44]. Copyright 2004.

brings about another important advantage: the QE of a thin QD layer is higher with respect to a thicker layer [42]. Therefore, this novel method opens up a new possibility for efficient white LED applications.

Förster-type nonradiative pumping of a color conversion layer is first proposed and theoretically investigated by Agranovich et al. [43]. As in the case of a direct dipole-dipole interaction, the energy transfer rate increases while the overlap integral between the emission of the donor and the absorption of the acceptor increases and it is also a highly distance dependent process, which is strong up to a distance of about $10 \mathrm{~nm}$. Afterwards, Achermann et al. showed the first experimental demonstration of energy-transfer pumping of semiconductor QDs using an epitaxial quantum well [44]. CdSe/ZnS core/shell QDs were hybridized on top of an InGaN/GaN quantum well with a thin GaN capping layer (ca. $2 \mathrm{~nm}$ ) using Langmuir-Blodgett technique (Fig. 16).
This nanostructure exhibited a FRET efficiency of $55 \%$ at room temperature. Thereafter, Rohrmoser et al. demonstrated the temperature dependence of exciton transfer from a single InGaN quantum well to colloidal CdS nanocrystal quantum dots. In this structure, the FRET efficiency is affected by two competing mechanisms: The increase of the temperature reduces the localization of excitons. On the other hand, as temperature increases nonradiative recombination channels within the quantum well start to be effective, which has an opposite effect on the energy transfer. Resultantly, an optimum transfer efficiency of $65 \%$ is obtained at $60 \mathrm{~K}$ [45]. Itskos et al. studied the distance dependence of the energy transfer process from a QW to a thin polyfluorene film (specifically poly(9,9-dioctylfluoreneco-9,9(di4-methoxyphenyl)fluorene) [46]. Nizamoglu et al. demonstrated white light generation by integrating redemitting QDs on a cyan-emitting wafer. In that work, the luminescence of the QDs in the hybrid system is raised by $63 \%$ with respect to the only QDs case as a result of strong FRET from QWs to QDs. The emitted warm white color is mapped to $(x, y)=(0.42,0.39)$ with a CCT of $3135 \mathrm{~K}$ [47]. However, one of the most important challenges for FRET utilization is its application to electrically driven LED devices.

An electrically pumped LED using nonradiative energy transfer has been demonstrated by Achermann et al. as shown in Fig. 17a [48]. For this system a single monolayer of $\mathrm{CdSe} / \mathrm{ZnS}$ core/shell nanocrystals has been integrated on an InGaN/GaN quantum well. The implementation of this hybrid device is difficult because of two conflicting factors. The rapid decrease of the FRET interaction with increasing QW-QD separation forces the use of very thin QW barrier layer (i.e., coupling is proportional to $d^{-4}$ ). However, if a very thin barrier layer is used, the current injection into the QW becomes a problem. As a solution to this problem, an inverted LED design is used such that a diode structure of $n-i-p$ is grown instead of a regular $p-i-n$ structure as shown in Fig. 17a. Since the $n$-doped GaN layer has a higher carrier mobility than the $p$-doped region, current injection is allowed despite a thin barrier layer of ca. $3 \mathrm{~nm}$. As illustrated in Fig. 17c, the emission of the QW at $420 \mathrm{~nm}$ and that of the QDs at $590 \mathrm{~nm}$ are clearly observable. The ratio between the $\mathrm{QD}$ and $\mathrm{QW}$ emission intensities is $13 \%$
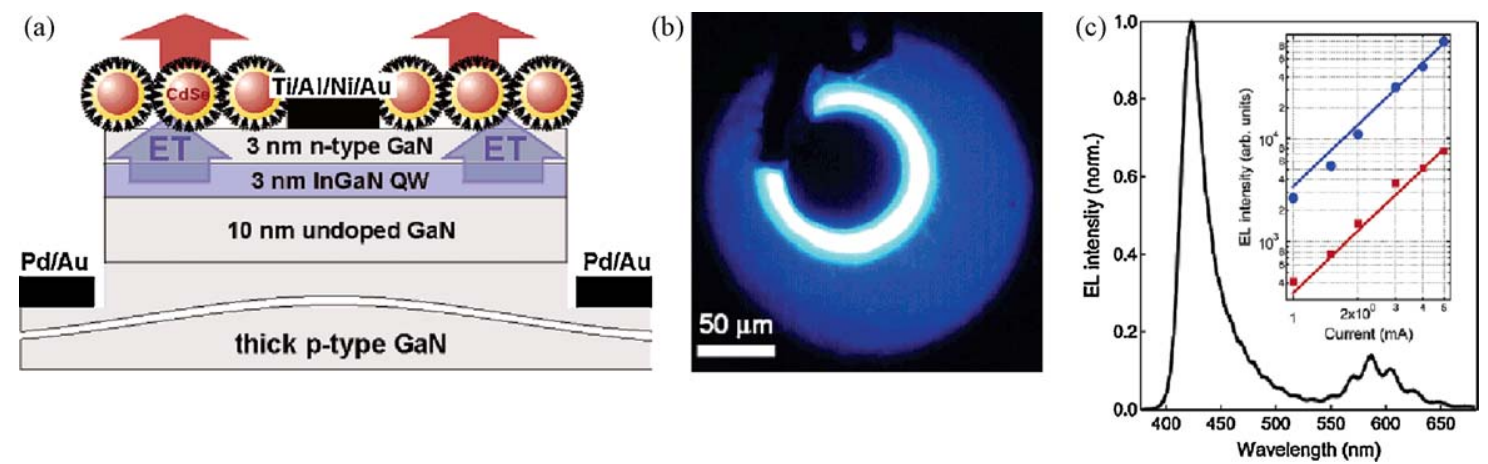

Figure 17 (a) Schematic cross section of the nonradiative energy transfer (ET)-LED structure, (b) LED under electrical excitation and (c) electroluminescence spectrum ( $2 \mathrm{~mA}$ driving current) displaying both QW $(420 \mathrm{~nm})$ and QD (590 nm) emission bands. Inset: QW (blue solid circles) and QD (red squares) emission intensities vs. driving current, indicating that the color-conversion efficiency (approximately the ratio of the two intensities) is independent of current [48]. Reprinted with permission from Reference [48]. Copyright 2006 American Chemical Society. 
which is independent on the applied current injection levels. The device color conversion efficiency corresponds to nearly $10 \%$. When compared to the case without FRET having a color conversion efficiency of $3.5 \%$, this is a significantly higher value. Therefore, FRET mediated excitonic color conversion is beneficial to achieve highly efficient LEDs.

Although Achermann et al. demonstrated an efficient way of color conversion, exciton transfer was limited. This is because only one quantum well could contribute to the color conversion through the nonradiative energy transfer process. As a solution, Chanyawadee et al. used an epiwafer having holes with elliptical cross-sections that reach down to the active QWs. This work uses LED structures following standard microfabrication and additional nanopatterning techniques including nanoimprint lithography as well as plasma etching such as inductively coupled reactive ion etching (ICP-RIE). The dimensions of the elliptic holes had large enough cross sections so that the drop-casted QDs can fill into the holes with a $450 \mathrm{~nm}$ depth. This architecture, which is referred to a deep-etched LED, is shown in Fig. 18 [49]. This design enables the QDs to be positioned in close proximity with multi-QWs so that excitons can be injected to the QDs. To compare with the deepetched LED, another LED has also been fabricated as shown in Fig. 18 (bottom). In this reference LED, the wafer is shallowly etched by $150 \mathrm{~nm}$ and the QDs are far from the multi-QW region to prohibit energy transfer. The disadvantage of the deep-etched LED is that its electroluminescence (EL) is not as strong as the shallow etched LED because of the defect states in the active layer introduced during etching. Deep-etched LED and shallow-etched LED have comparable EL at $7 \mathrm{~mA}$ and $3.8 \mathrm{~mA}$, respectively (Fig. 19). However, for the same EL intensity of QW emission, the QD emission of the deep-etched LED is higher than the shallowetched LED because of the additional exciton transfer from QWs to QDs. The color conversion efficiency of the deepetched LED is $20 \%$, which is $43 \%$ times higher than that of the shallow-etched LED, doubling the value reported by Achermann et al. [48]. With this structure an energy transfer efficiency of $82 \%$ is achieved, which is significantly higher
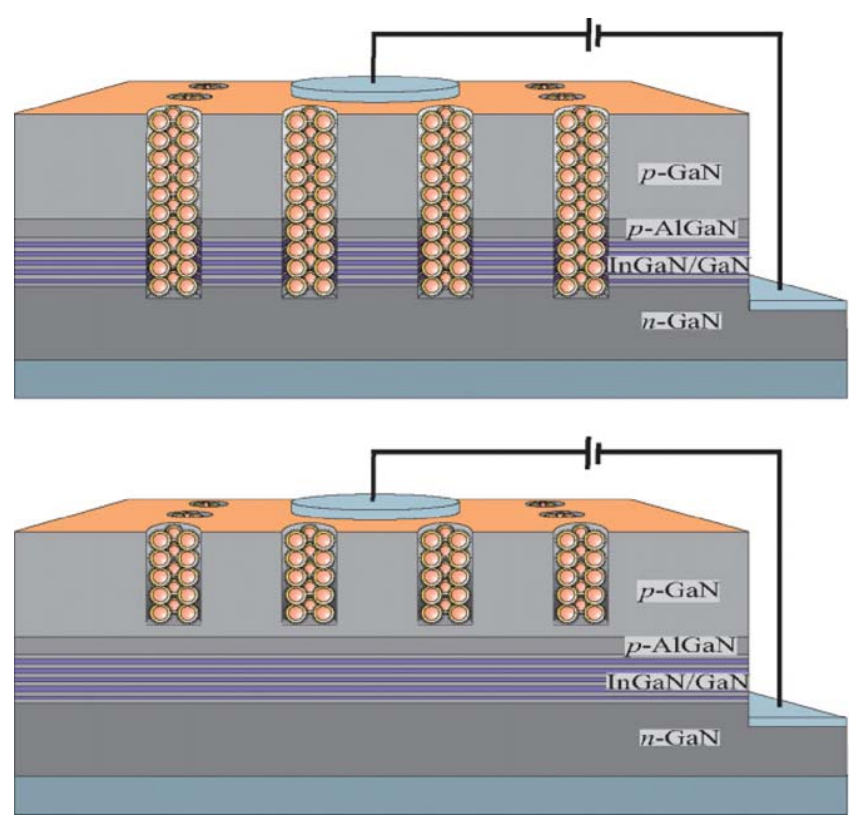

Figure 18 Schematic diagrams of the hybrid QD-LED etched to different levels: through the MQW (top) and only down to the p-AlGaN layer (bottom). Reprinted with permission from Reference [49]. Copyright 2010 WILEY-VCH Verlag GmbH \& Co. KgaA, Weinham.

than the previously reported levels for QW-QD systems. However, just $18 \%$ of the generated excitons experienced such a high energy transfer efficiency. To make efficient LEDs, the ratio of excitons transferred to QDs needs to be increased. Therefore, novel system architectures are necessary to increase the exciton transfer ratio while achieving high energy transfer efficiency. Here, it is also worth mentioning that the illustration in Fig. 18 presents an idealized case where the holes are completely and neatly stuffed with QDs. The variations in this QD distribution might be expected to affect the final FRET efficiency of the material system.
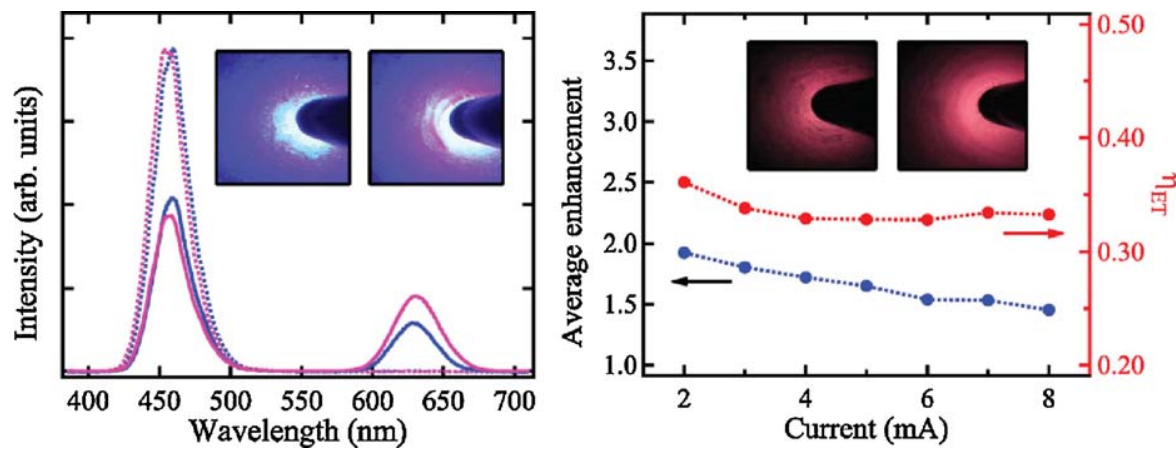

Figure 19 QD emission enhancement and energy-transfer efficiency of the hybrid color-conversion LED. (a) QW emission of the bare shallow-etched LED (dotted blue line) and the bare deep-etched LED (dotted pink line) at 3.8 and $7 \mathrm{~mA}$, respectively. The solid blue line (solid pink line) represents the corresponding EL of the hybrid QD/shallow-etched LED (hybrid QD/deep-etched LED). Inset: left (right) panel shows emission of the hybrid shallow etched (deep-etched) LED for injection currents that produce the same QW emission intensity in the absence of QDs. (b) Red solid circles represent the effective nonradiative energy transfer efficiency versus the deep-etched LED injection current. Blue solid circles represent the enhancement of the QD emission. Inset: left (right) panel shows the emission of the QDs only in the hybrid shallow-etched (deep-etched) LED. Reprinted with permission from Reference [49]. Copyright 2010 WILEY-VCH Verlag GmbH \& Co. KgaA, Weinham. 

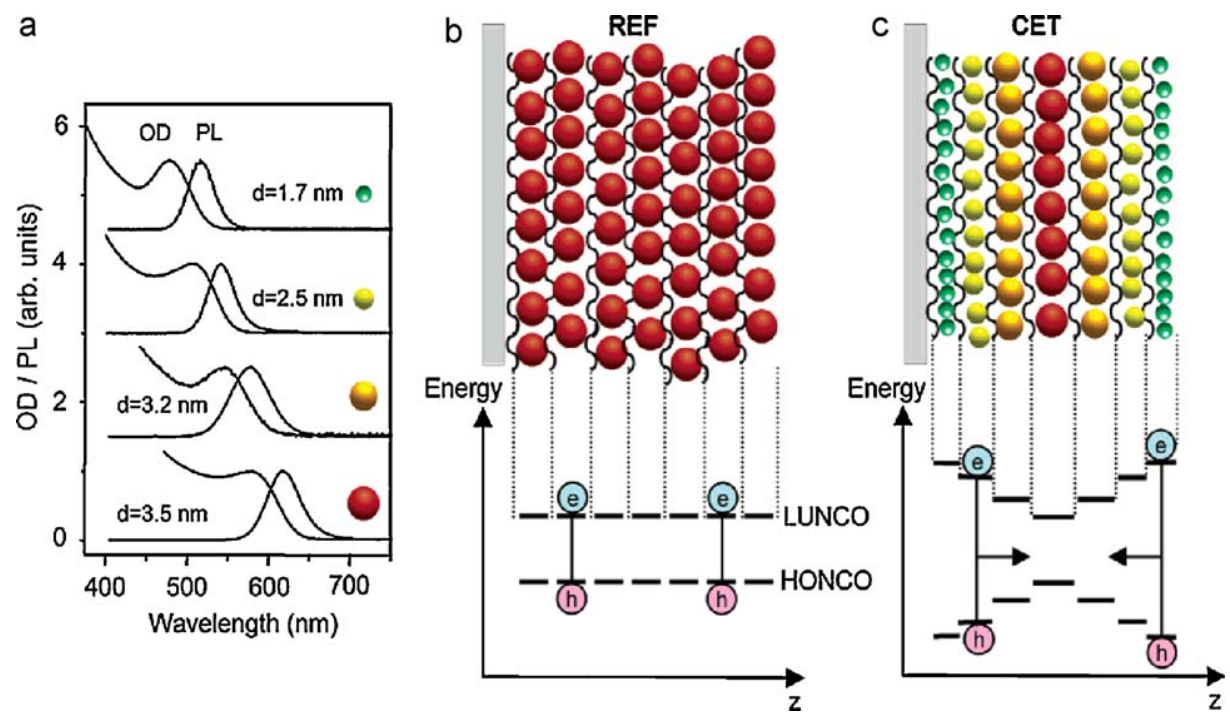

Figure 20 (a) Absorbance (optical density, OD) and photoluminescence (PL) spectra of the CdTe QDs of four different sizes used to prepare the layer-by-layer assembled samples. The sizes are chosen such that the emission spectrally matches the excitonic absorption of subsequently larger QDs. All spectra are taken in aqueous solution and vertically shifted for clarity. (b) Schematic sketch of the REF sample, which consists of seven layers of red-emitting, 3.5-nm large CdTe QDs. For the REF sample the energetic positions of the highest occupied nanocrystal orbital (HONCO) and the lowest unoccupied nanocrystal orbital (LUNCO) are sketched. Each excitation remains in the layer where it is created because of the absence of a gap gradient. (c) Sample CET consists of subsequent layers comprising green, yellow, orange, red, orange, yellow, and green-emitting QDs. For the CET sample the HONCO and LUNCO are also sketched, visualizing the cascaded band gaps used to facilitate cascaded energy transfer [50]. Reprinted with permission from Reference [50]. Copyright 2004 American Chemical Society.

\section{FRET-enhanced LEDs}

QDs usually exhibit lower quantum efficiencies in films as compared to their in-solution quantum efficiencies. As a possible solution, higher band gap inorganic capping shells are used but these shell barriers also do not necessarily perfectly isolate the QDs unless they are thick enough. In a recent study Franzl et al. showed a cascaded energy transfer structure made of QDs with funnel-like band gap profiles and demonstrated that the funnel structure recycles trapped and otherwise lost electron-hole pairs increasing the emission intensity [50]. In that work, CdTe QDs were synthesized in water with a thioglycolic acid as short-chain capping agent. Positively charged poly[diallyldimethylammonium chloride] (PDDA) was used as a polyelectrolyte to assemble the QDs in a layer-by-layer architecture with a funnel-like band gap profile. CdTe QDs with four different diameters between 1.7 and $3.5 \mathrm{~nm}$ emitting green, yellow, orange and red colors were utilized (Fig. 20a). As a result of this size variation, a spectral overlap occurs between the emission of the QDs with smaller diameter and the absorption of the QDs with the larger diameter which is one of the necessary conditions for FRET. In-solution quantum efficiencies of the samples were in the range of $8-34 \%$. For the reference sample, seven layers of red-emitting QDs were deposited as shown in Fig. 20b (for which the sample is named as REF). For a funnel-like band gap profile, subsequent layers of green-, yellow-, orange-, red-, orange-, yellow-, and green- emitting QDs are deposited as indicated in Fig. 20c (where the sample is dubbed CET). The quantum efficiency obtained for the REF sample was ca. $2 \%$, which is much lower with respect to the QEs of the QDs in solution. In other words, in this QD film about $98 \%$ of the excited electron-hole pairs do not experience interband recombination and the trapped excitons recombine through midgap defect states. However, it was shown that the CET sample exhibited four-fold enhanced red color emission than the REF sample, although the REF sample contains seven times more red-emitting QDs. By using such strong FRET processes, trapped excitons can be recycled and can be used to enhance the emission of color conversion layers.

For LED applications, QDs are hybridized as a mixture of QDs and polymer matrix, or a mixture of QDs and an organic capping layer on top of the QD layer to isolate the QDs. The quantum efficiency enhancement in nanocrystal solids is critically important for their efficient use as luminophors on color-conversion LEDs. Furthermore, recycling of trapped excitons reveals emission increase in the QD layer. Thus, in a recent study, the optimum QE enhancement for QD close-packed films was explored for LED applications [51]. The QE enhancement was investigated as a function of donor-acceptor QD ratios as shown in Fig. 21. The authors used cyan-emitting CdSe/ZnS core/shell QDs as the donors and green-emitting QDs as the acceptors with spectral overlap and dot-dot interspacing satisfying the necessary conditions for efficient FRET. The control groups of samples consisting of only cyan- and of only green-emitting QDs were used as the base line and these points are presented at the far edges of the plot in Fig. 21 for visualization purposes. All of the cyan- and green-emitting QD hybrids exhibit quantum efficiency enhancement because of the recycling of trapped excitons. A maximum quantum efficiency enhancement of 


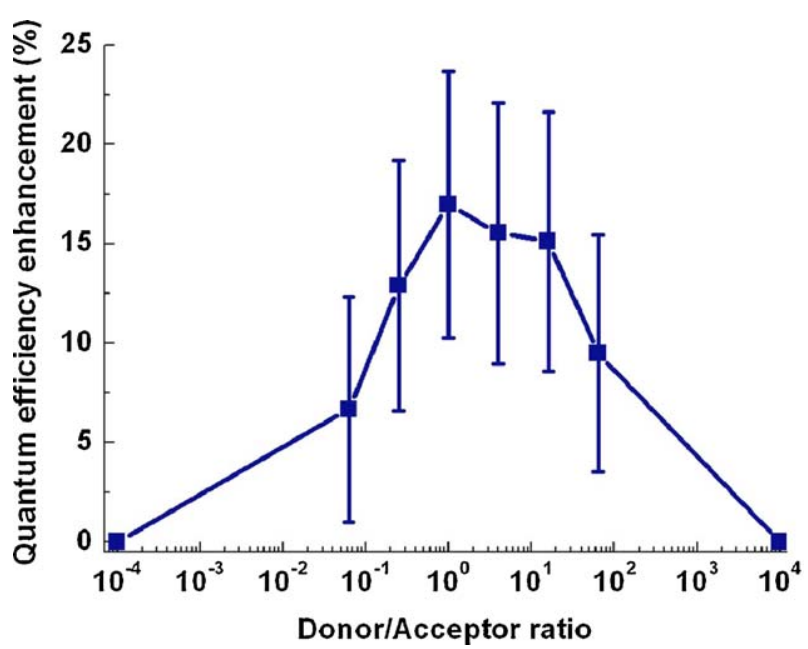

Figure $21 \quad$ QD donor-acceptor ratio dependent quantum efficiency enhancement of the donor-acceptor hybrid solid films consisting of cyan- and green-emitting CdSe/ZnS core/shell QDs. Reprinted with permission from Reference [51]. Copyright 2009 American Institute of Physics.

$17 \%$ is achieved when the donor-acceptor ratio is $1: 1$ while having a FRET efficiency of $48 \%$.

The mixture of green- and yellow-emitting QDs with such an optimized donor-acceptor ratio was integrated on a near-UV LED (Fig. 22) [52]. The QE of this energy gradient structure was found to be $13.2 \%$ higher compared to the corresponding control groups which is again a consequence of the recycling of trapped excitons. The violet tail of the nearUV emission balanced out the green and yellow emission for white light generation and the operating point of the hybrid LED corresponded to $(x, y)=(0.44,0.40)$ and a CCT $=2872 \mathrm{~K}$. This work demonstrates enhanced white light generation via FRET and showcases the first application of FRET enhanced white LEDs.

Similarly, this idea may be also applied to green LEDs to remedy the green-gap problem [3]. Indeed, integration

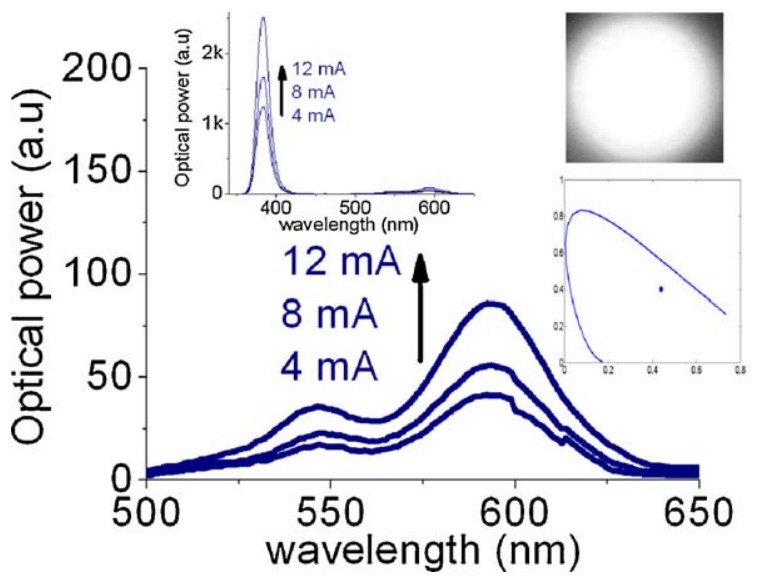

Figure 22 Luminescence spectra of mixed green and redemitting $\mathrm{CdSe} / \mathrm{ZnS}$ core/shell nanocrystals integrated on a near-UV InGaN/GaN LED. Insets show the evolution of the optical power with increasing current injection, a true color image of the hybrid LED, and its $(x, y)$ tristimulus coordinates [52]. of QD layers with an energy gradient towards green emission on an efficient near-UV LED platform ends in a $19.5 \%$ QE enhancement of a QD layer whose QE reaches to $55 \%$ [53].

\section{Future outlooks and conclusion}

In conclusion, here we reviewed the recent developments regarding QD-integrated light emitting diodes, which are based on the color conversion phenomenon, after a brief introduction to color science, photometry and optical properties of QDs. As a concluding remark to the discussed issues, we can add that the most promising and attractive features of colloidal QDs for color conversion applications are their narrow but precisely tuneable emission spectra, their very high photoluminescence quantum yields, their stability inherent to inorganic substances as well as the possibility to adjust their surface functionality, which allows literally unlimited design of functional composites and hybrid structures. Considering these advantages, QDs offer a very significant contribution to this developing field in order that the future forecasts regarding the replacement of traditional light sources by light emitting diodes can be realized. Especially, the broad emission spectra problem of phosphors, and cost and green gap issues of multichip LED approaches can be by-passed by the integration of QDs into light emitting diodes; as a result, the targeted performance levels of $200-300 \mathrm{~lm} / W_{\text {elect }}$ can be realized in the near future [54]. Since the large scale QD synthesis has been succeeded in recent years [55], achieving the mass production of QD-LEDs with such an extraordinary performance might be possible. However, there are still some aspects of QDs that need further development. One of these challenges is the reduction of the potential toxicity of quantum dots, as the most successful examples of this kind of materials are cadmium-based. Existing alternatives such as InP or ZnTe QDs suffer from relatively low stability and moderate quantum yields. Therefore, more research in the direction of improving these material systems or other possible candidates is to be needed. Another important challenge is the compatibility of QDs with the silicone matrix typically used for the fabrication of conversion layers in the LED industry. For solving this problem, a proper surface design and protecting shells are necessary so that the QDs are dispersed in silicone without any deterioration of the photoluminescence and stability. As an alternative to the silicone, the exploration of other polymeric candidates, which are compatible with QDs, can be proposed as an important research subject that will open new possibilities for the use of QDs in LEDs. Despite these difficulties, some companies such as Evident Technologies and Nanosys Inc. had already introduced their QD integrated LEDs, though not yet for large-scale general lighting. In case these challenges are overcome, the quantum dots should be expected to attain a dominant place in the lighting industry within one or two decades.

\section{Acknowledgement}

This work is supported by National Research Foundation under Grant No. NRF-RF-2009-09 and NRF-CRP-6-2010-2, 
and also by EU-FP7 Nanophotonics4Energy NoE, BMBF TUR 09/001, and TUBITAK EEEAG 109E002, 109E004, 110E010, and 110E217. H.V.D. acknowledges support from ESF-EURYI and TUBA-GEBIP.

\section{References}

[1] Renewable Energy Policy Network for 21st Century, "Renewables 2010 Global Status Report," 2010.

[2] D. Irvine-Halliday, R. Peon, G. Doluweera, I. Platonova, G. Irvine-Halliday, SPIE Newsroom (2006).

[3] http://www.wind-energie.de/de/statistiken (Accessed on November 21, 2010).

[4] D.J.C. MacKay, Sustainable Energy - Without The Hot Air, 1st ed., UIT Cambridge Ltd., Cambridge, 2009.

[5] E.F. Schubert, Light-Emitting Diodes, 2nd ed., University Press, Cambridge, Cambridge, 2006.

[6] J.Y. Tsao, IEEE Circ. Dev. Mag. 20 (2004) 28-37.

[7] O. Graydon, Nat. Photon 5 (2011) 1.

[8] J.J. Vos, Color Res. Appl. 3 (1978) 125-128.

[9] CIE, Commission Internationale de l'Eclairage Proceedings, Cambridge University Press, Cambridge, 1931.

[10] A. Stockman, in: T.G. Brown, K. Creath, H. Kogelnik, M.A. Kriss, J. Schmit, M.J. Weber (Eds.), The Optics Encyclopedia: Basic Foundations and Practical Applications, vol. 1, WileyVCH, Berlin, 2004, pp. 207-226.

[11] G. Wyszecki, W.S. Stiles, Color Science Concepts and Methods, Quantitative Data and Formulae, 2nd ed., John Wiley and Sons, New York, 2000.

[12] CIE, Method of Measuring and Specifying Color-Rendering of Light Sources. CIE publication No. 13.3., CIE, Vienna, Austria, 1995.

[13] Y. Ohno, Proc. SPIE 5530 (2004) 88-98.

[14] Y. Ohno, Opt. Eng. 44 (2005) 111302.

[15] W. Davis, Y. Ohno, Proc. SPIE 5941 (2005) $59411 \mathrm{G}$.

[16] M.R. Krames, O.B. Shchekin, R. Mueller-Mach, G. Mueller, L. Zhou, G. Harbers, et al., J. Display Technol. 3 (2007) 160-175.

[17] C.B. Murray, D.J. Norris, M.G. Bawendi, J. Am. Chem. Soc. 115 (1993) 8706-8715.

[18] C.D.M. Donega, P. Liljeroth, D. Vanmaekelbergh, Small 1 (2005) 1152-1162.

[19] A.L. Rogach, T. Franzl, T.A. Klar, J. Feldmann, N. Gaponik, V. Lesnyak, et al., J. Phys. Chem. C 111 (2007) $14628-14637$.

[20] N. Gaponik, A.L. Rogach, Phys. Chem. Chem. Phys. 12 (2010) 8685-8693.

[21] N. Gaponik, A. Rogach, in: A.L. Rogach (Ed.), Semiconductor Nanocrystal Quantum Dots, Springer-Verlag, Wien, 2008, pp. 73-99.

[22] N. Gaponik, S.G. Hickey, D. Dorfs, A.L. Rogach, A. Eychmüller, Small 6 (2010) 1364-1378.

[23] D.V. Talapin, J.-S. Lee, M.V. Kovalenko, E.V. Shevchenko, Chem. Rev. 110 (2010) 389-458.

[24] N. Gaponik, J. Mater. Chem. 20 (2010) 5174-5181.

[25] S. Nizamoglu, G. Zengin, H.V. Demir, Appl. Phys. Lett. 92 (2008) 031102.

[26] T. Erdem, S. Nizamoglu, X.W. Sun, H.V. Demir, Opt. Express 18 (2010) 340-347.

[27] J. Lee, V.C. Sundar, J.R. Heine, M.G. Bawendi, K.F. Jensen, Adv. Mater. 12 (2000) 1005-1102.

[28] S. Nizamoglu, T. Ozel, E. Sari, H.V. Demir, Nanotechnology 18 (2007) 065709.

[29] H.-S. Chen, C.-K. Hsu, H.-Y. Hong, IEEE Photon. Technol. Lett. 18 (2006) 193-195.

[30] Solid State Lighting, Sandia National Laboratories, http: / /lighting.sandia.gov (Accessed on October 7, 2011).
[31] E. Jang, S. Jun, H. Jang, J. Lim, B. Kim, Y. Kim, Adv. Mater. 22 (2010) 3076-3080.

[32] H.-S. Chen, D.-M. Yeh, C.-F. Lu, C.-F. Huang, W.-Y. Shiao, J.-J. Huang, et al., IEEE Photon. Technol. Lett. 18 (2006) $1430-1432$.

[33] S. Nizamoglu, E. Mutlugun, T. Ozel, H.V. Demir, S. Sapra, N. Gaponik, et al., Appl. Phys. Lett. 92 (2008) 113110.

[34] S. Sapra, S. Mayilo, T.A. Klar, A.L. Rogach, J. Feldmann, Adv. Mater. 19 (2007) 569-572.

[35] S. Nizamoglu, E. Mutlugun, O. Akyuz, N.K. Perkgoz, H.V. Demir, L. Liebscher, et al., New J. Phys. 10 (2008) 023026.

[36] J.D. Gosnell, S.J. Rosenthal, S.M. Weiss, IEEE Photon. Technol. Lett. 22 (2010) 541-543.

[37] J.H. Ahn, C. Bertoni, S. Dunn, C. Wang, D.V. Talapin, N. Gaponik, et al., Nanotechnology 18 (2007) 335202.

[38] H.V. Demir, S. Nizamoglu, T. Ozel, E. Mutlugun, I.O. Huyal, E. Sari, et al., New J. Phys. 9 (2007) 362.

[39] H.S. Jang, H. Yang, S.W. Kim, J.Y. Han, S.-G. Lee, D.Y. Jeon, Adv. Mater. 20 (2008) 2696-2702.

[40] H.S. Jang, B.-H. Kwon, H. Yang, D.Y. Jeon, Appl. Phys. Lett. 95 (2009) 161901.

[41] J. Ziegler, S. Xu, E. Kucur, F. Meister, M. Batentschuk, F. Gindele, et al., Adv. Mater. 20 (2008) 4068-4073.

[42] Q. Dai, C.E. Duty, M.Z. Hu, Small 6 (2010) 1577-1588.

[43] D. Basko, G.C. La Rocca, F. Bassani, V.M. Agranovich, Eur. Phys. J. B 8 (1999) 353-362.

[44] M. Achermann, M.A. Petruska, S. Kos, D.L. Smith, D.D. Koleske, V.I. Klimov, Nature 429 (2004) 642-646.

[45] S. Rohrmoser, J. Baldauf, R.T. Harley, P.G. Lagoudakis, S. Sapra, A. Eychmüller, et al., Appl. Phys. Lett. 91 (2007) 092126.

[46] G. Itskos, G. Heliotis, P.G. Lagoudakis, J. Lupton, N.P. Barradas, E. Alves, et al., Phys. Rev. B 76 (2007) 035344.

[47] S. Nizamoglu, E. Sari, J.-H. Baek, I.-H. Lee, H.V. Demir, New J. Phys. 10 (2008) 123001.

[48] M. Achermann, M.A. Petruska, D.D. Koleske, M.H. Crawford, V.I. Klimov, Nano Lett. 6 (2006) 1396-1400.

[49] S. Chanyawadee, P.G. Lagoudakis, R.T. Harley, M.D.B. Charlton, D.V. Talapin, H.W. Huang, et al., Adv. Mater. 22 (2010) 602-606.

[50] T. Franzl, T.A. Klar, S. Schietinger, A.L. Rogach, J. Feldmann, Nano Lett. 4 (2004) 1599-1603.

[51] S. Nizamoglu, O. Akin, H.V. Demir, Appl. Phys. Lett. 94 (2009) 243107.

[52] S. Nizamoglu, H.V. Demir, Opt. Express 16 (2008) 13961-13968.

[53] S. Nizamoglu, E. Sari, J.-H. Baek, I.-H. Lee, H.V. Demir, IEEE J. Sel. Top. Quant. Electron 15 (2009) 1163-1170.

[54] R. Haitz, J.Y. Tsao, Phys. Status Solid. A 208 (2011) $17-29$.

[55] K. Sanderson, Nature 459 (2009) 760-761.

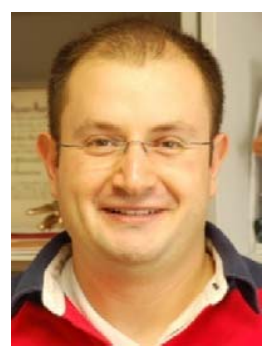

Hilmi Volkan Demir is NRF fellow of Singapore and Nanyang Associate Professor and Director of Luminous! Center of Excellence for Semiconductor Lighting and Displays at NTU. Concurrently, he is EURYI Associate Professor of Electrical Engineering and Physics, Bilkent University and UNAM-National Nanotechnology Research Center. Demir earned Ph.D. (2004) and M.Sc. (2000) degrees from Stanford University, and B.Sc. (1998) from Bilkent. Demir's research has contributed to commercialization of several new enabling technologies and $>20$ patent applications and several important awards/programs including TUBITAK-TESVIK and NRF-CRP. Demir published $>90$ SCl-papers and delivered $>150$ invited seminars on solid-state lighting and nanoparticles/nanocrystals research in industry and academia. 


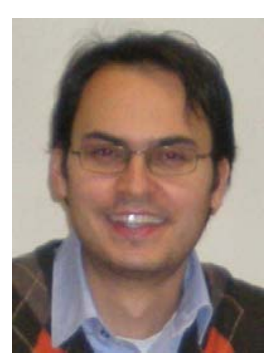

Dr. Sedat Nizamoglu received his Ph.D. degree in electrical and electronics engineering in 2011 following his M.Sc. degree in Physics in 2007 and his B.Sc. degree in Electrical and Electronics Engineering in 2005, both in Prof. Hilmi Volkan Demir's group at Bilkent University. He has published 29 papers in indexed journals and is a co-inventor of two pending patents. He won several awards including the Student Innovation Award given by the European Technology Platform Photonics21, IEEE Photonics Society Graduate Student Fellowship, and the SPIE Scholarship Award in Optical Science and Engineering. He is currently a Postdoctoral Fellow at Harvard University.

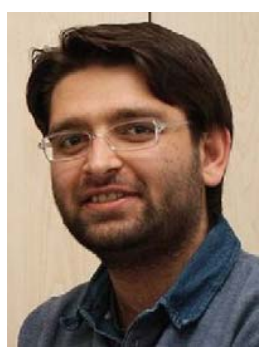

Talha Erdem received his B.Sc. degree in 2009 and M.Sc. degree in 2011 both in electrical and electronics engineering from Bilkent University. He is currently studying towards his Ph.D. degree in the group of Prof. Hilmi Volkan Demir. He is the coauthor of $5 \mathrm{SCl}$ paper. His research is focused on the design and implementation of highquality, quantum-dot-integrated white LEDs and related structures.

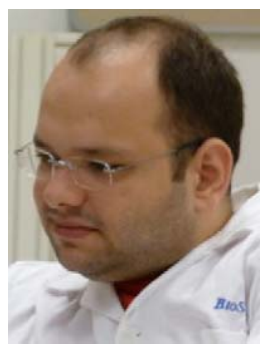

Evren Mutlugun received his BSc degree in 2005 from Middle East Technical University Physics Department, Ankara, Turkey. In the research group of Prof. Hilmi Volkan Demir, he completed his MSc studies in Bilkent University Physics Department in 2007 and, since then, he has been working towards the completion of his Ph.D. degree in the same department. He has over 15 papers in $\mathrm{SCl}$ journals and is the co-inventor of two patents. $\mathrm{He}$ has been awarded with 2010 SPIE Graduate Scholarship in Optical Science and Engineering.

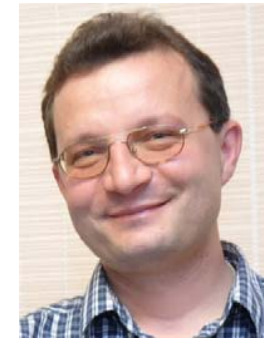

Nikolai Gaponik is a senior staff scientist at the Technical University of Dresden. He received his Ph.D. in chemistry (2000) from the Belarusian State University in Minsk, studying electrochemical and photoelectrochemical properties of composite materials based on conducting polymers and semiconductor nanocrystals. He was a visiting scientist at the Ludwig-MaximiliansUniversität in Munich in 2000, a DAAD-fellow and a research scientist in the group of Prof. $\mathrm{H}$. Weller at the University of Hamburg in 2000-2005 and a IKERBASQUE fellow at the University of Basque Country in San Sebastian in 2011. His current research focuses on the synthesis, assembly and applications of colloidal semiconductor and metal nanocrystals.

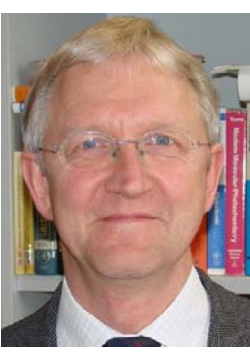

Alexander Eychmüller obtained his Ph.D. (1987) working on proton transfer reactions at the MPI for Biophysical Chemistry in Göttingen under the supervision of Dr. K.-H. Grellmann and Prof. A. Weller. After a postdoctoral year at UCLA working with Prof. M. A. El-Sayed he joined the group of Prof. A. Henglein at the $\mathrm{HMI}$ in Berlin. In 1994 he moved with Prof. $\mathrm{H}$. Weller to the University of Hamburg focusing his scientific interests on the photophysical and structural properties of semiconductor nanocrystals. The habilitation in 1999 and the venia legendi in 2000 were followed by an offer of a chair in Physical Chemistry/Electrochemistry of the TU Dresden in 2005. 